\title{
Nanoscale Advances
}

Check for updates

Cite this: Nanoscale Adv., 2021, 3, 963

Received 25th November 2020 Accepted 31st December 2020

DOI: 10.1039/dOna00987c

rsc.li/nanoscale-advances

\section{Emerging field of few-layered intercalated 2D materials $\uparrow$}

\author{
Qing Cao, (D) Fabian Grote, (D) Marleen Hußmann (D) and Siegfried Eigler (D)*
}

The chemistry and physics of intercalated layered 2D materials (2DMs) are the focus of this review article. Special attention is given to intercalated bilayer and few-layer systems. Thereby, intercalated few-layers of graphene and transition metal dichalcogenides play the major role; however, also other intercalated 2DMs develop fascinating properties with thinning down. Here, we briefly introduce the historical background of intercalation and explain concepts, which become relevent with intercalating few-layers. Then, we describe various synthetic methods to yield intercalated 2DMs and focus next on current research directions, which are superconductivity, band gap tuning, magnetism, optical properties, energy storage and chemical reactions. We focus on major breakthroughs in all introduced sections and give an outlook to this emerging field of research.

\section{Introduction}

Our mobile and rechargeable world is based on intercalation chemistry. Thus, it is not surprising that the Nobel Prize in Chemistry 2019 was given to the pioneers of the Li-ion battery, Goodenough, Whittingham, and Yoshino. ${ }^{1}$ The pioneering work of Whittingham was the report on the reversible intercalation of layered $\mathrm{TiS}_{2}$ by Li-ions. The work of Goodenough led to the discovery of layered cobalt oxide as cathode material and the work of Yoshino explored graphite as anode material. Now, energy can be stored by collecting photons from the sun or collecting kinetic energy from wind or water and used at will.

Institute of Chemistry and Biochemistry, Freie Universität Berlin, Takustraße 3, 14195 Berlin, Germany.E-mail: siegfried.eigler@fu-berlin.de

$\dagger$ Dedicated to Professor Andreas Hirsch on the occasion of his 60th birthday.
With the discovery of graphene by Geim and Novoselov, which was awarded with a Nobel Prize in 2010 it was revealed that properties of materials change with thinning down to the single layer. ${ }^{2}$ Thus, novel materials emerged and were studied by physicists. In addition, the chemistry of $2 \mathrm{D}$ materials (2DMs) came into the focus of research and new functionalization principles were developed, both of covalent and non-covalent nature. ${ }^{3-5}$ Surface physics and surface chemistry, both accelerated in recent years and thus, researchers realized that 2DMs properties can be tailored by surface modification, such as the deposition of metal particles or organic donor and acceptor type molecules, respectively. ${ }^{6,7}$ In general, countless investigations are being made into the chemistry and physics of 2DMs and a practically infinite variety is emerging. Thereby, transition metal dichalcogenides (TMDCs) or 2D allotropes of $\mathrm{Si}, \mathrm{Bi}, \mathrm{P}$ and others possess different band structures. Thus, 2DMs are insulators, topological insulators, semiconductors, conductors,

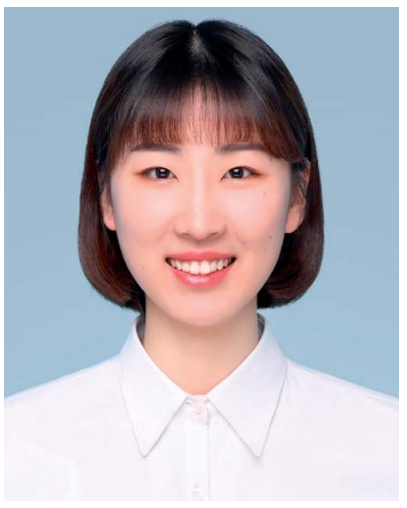

materials.
Qing Cao received her $\mathrm{MSc}$ from the Institute of Advanced Materials at Nanjing Tech University in 2019. She received her BSc in polymer materials from Shandong University of Science and Technology in 2016. She is currently a doctoral candidate in the research group of Prof. Siegfried Eigler at Freie Universität Berlin. Her research mainly focuses on intercalation and functionalization of $2 D$

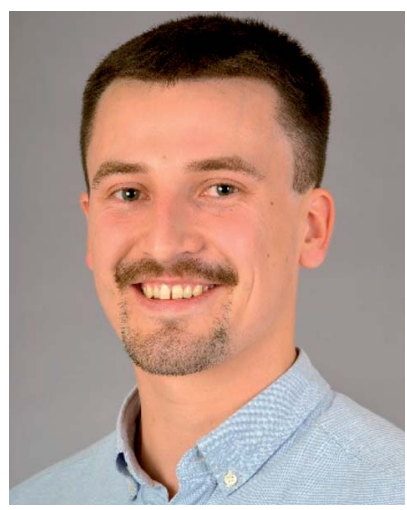

Fabian Grote is a doctoral candidate in the group of Prof. Siegfried Eigler at Freie Universität Berlin. He received his $\mathrm{MSc}$ in chemistry from the Julius-Maximilians-Universität Würzburg in 2019 and his BSc in chemistry from the FriedrichAlexander-Universität ErlangenNürnberg in 2016. His research focuses on the chemistry and fundamental properties of oxofunctionalized graphene. 
semimetals or semiconductors with direct or indirect bandgap. ${ }^{8}$ In recent years, research on the fabrication of heterostructures is targeted by artificially assembling those 2DMs with combining and extending physical properties. ${ }^{9}$

Historically, the term intercalation and intercalation chemistry was coined by Rüdorff and Hoffmann in $1938,{ }^{10}$ although intercalation in the form of swelling was already observed in 1841 by Schafhaeutl. ${ }^{\mathbf{1 1}}$ Key-results of graphite intercalation compounds (GICs) are summarized and explained by M. S. Dresselhaus and G. Dresselhaus. ${ }^{12}$ Thereby, intercalation is performed in bulk materials and various GICs are described, which are divided into acceptor and donor type GICs, respectively. Examples are graphite sulfate and potassium graphite, which are the best studied materials so far. ${ }^{12}$

However, in light of recent developments, it is more obvious than ever that the intercalation of stacks of 2DMs is of particular interest. While a single layer of a 2DM cannot be intercalated, bilayers (2Ls) and few-layers can already be intercalated, as summarized in this review. As known from the experience of graphene physics compared to graphite physics, the properties of single- and few-layered 2DMs differ from the bulk e.g. due to confinement of charge carriers. ${ }^{13}$ Because of the ease of language, the expression "intercalation of $2 \mathrm{DMs}$ " is used in this article for the intercalation of any few-layered 2DMs. In addition, we propose to expand the terminology of intercalation, which should not be limited to the traditional process in which atoms or ions penetrate the galleries of layered compounds. In this review we use the term "intercalated" to describe the final product, which can be regarded as an intercalated layered system. Accordingly, a 2DM may be intercalated between molecular layers of e.g. organic molecules. In this example surface science and intercalation mix. A single layer of a 2DM may be intercalated between other 2DMs, such as graphene encapsulated between hBN layers, e.g. by artificial assembly, a structure which can also be termed as van der Waals (vdW) structure. ${ }^{\mathbf{1 4}}$ With this expanded view of intercalation, a huge research area can be identified and summarized.
A)

B)

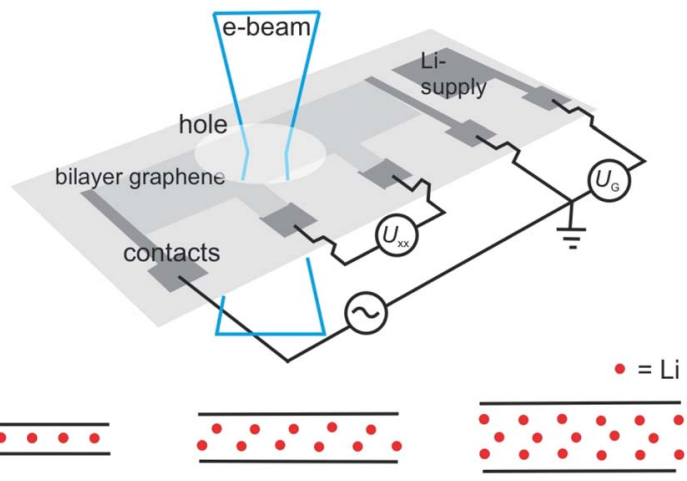

Fig. 1 Illustration of in situ transmission electron microscopy of the Liintercalation process in suspended $2 \mathrm{~L}$ graphene, triggered in a device. (A) Illustration of the device on a $\mathrm{Si}_{3} \mathrm{~N}_{4}$-covered Si substrate. (B) from left to right: side-views of expected 'conventional' $\mathrm{C}_{6} \mathrm{LiC}_{6}$ configuration and of the experiment matching structures with $2 \mathrm{~L} \mathrm{Li} \mathrm{crystal} \mathrm{and}$ $3 \mathrm{~L} \mathrm{Li} \mathrm{crystal.}{ }^{15}$

As a motivating and fascinating recent example, we want to highlight the intercalation of $2 \mathrm{~L}$ graphene by $\mathrm{Li}$, a process which is related to the anode process of a Li-ion battery. ${ }^{15}$ A $2 \mathrm{~L}$ of graphene was mounted on a grid in the SALVE microscope (sub-Angstrom-low-voltage-electron microscope) and the intercalation was triggered by a $5 \mathrm{~V}$ potential (Fig. 1A). As depicted in Fig. 1, the process of intercalation can be followed microscopically with atomic resolution. The growth of the Li crystal between $2 \mathrm{~L}$ graphene was conducted and filtering the structure of the $2 \mathrm{~L}$ graphene led to the conclusion that the Li crystal differs from the expected packing for $\mathrm{C}_{6} \mathrm{LiC}_{6}$ (Fig. 1B), but can be explained by the formation of a $2 \mathrm{~L}$ and a trilayer $(3 \mathrm{~L})$ of $\mathrm{Li}$. Thus, those results differ from bulk lithium graphite with the formular $\mathrm{LiC}_{6}$, since more $\mathrm{Li}$ atoms can be intercalated between two layers compared to the bulk.

Other review articles and books are available, however summarizing primarily the intercalation of bulk materials. In particular the review by Stark et al. summarizes intercalation

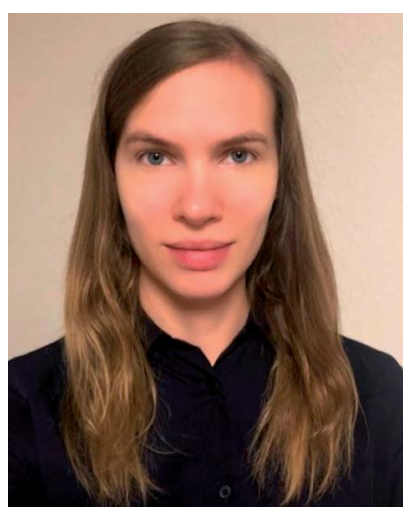

Marleen Hußmann received her BSc as well as her MSc in chemistry from Freie Universität Berlin in 2016 and 2019, respectively. She is currently a doctoral candidate in the group of Prof. Siegfried Eigler with the research topic of photoluminescence analysis and construction of heterostructures including molecules.

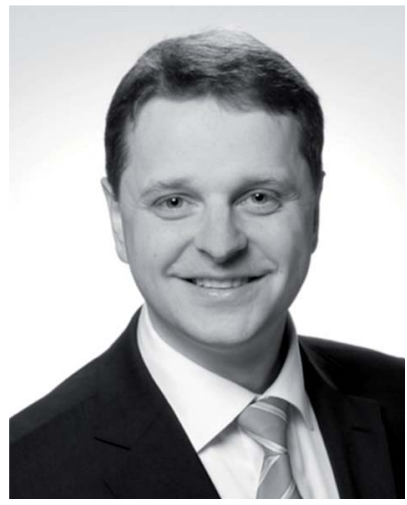

Siegfried Eigler received his $P h D$ in organic chemistry from the Friedrich-Alexander-Universität Erlangen-Nürnberg in 2006. Subsequently, he conducted basic research on electrically conductive polymers and graphene oxide as an industry chemist. In 2016 he accomplished habilitation at Friedrich-Alexander-Universität Erlangen-Nürnberg and became Associate Professor at the Chalmers University of Technology. Now, he is Professor at Freie Universität Berlin and his research focuses on the controlled chemistry of graphene, synthesis and evaluation of fluorophores and fabrication of devices. 
processes not only on bulk, but also $2 \mathrm{D}$ level. ${ }^{12,16-20}$ Thus, here we keep history and bulk information short, since it is covered in the above mentioned articles. Further, we introduce important principles relevant for few-layer, trilayer $(3 \mathrm{~L})$, bilayer $(2 \mathrm{~L})$ and monolayer (1L) intercalation. Moreover, we highlight the recent advances. More specifically, we will focus on concepts, the synthetic access to intercalated 2DMs and introduce common methods, such as vapor transport, solvent based or electrochemical methods and artificial assembly. Next, we highlight recent results and properties of intercalated 2DM systems.

\section{Concept of staging}

Here, we introduce concepts and definitions relevant for 2DMs intercalation, which are derived from definitions introduced for GICs. We note that the historical background and intercalation concepts for bulk materials are summarized in the excellent perspective article of Lerf. ${ }^{20}$ In particular, staging and charge transfer are of importance to describe the structure and properties of intercalation compounds. With thinning down the bulk to the $1 \mathrm{~L}$ additional considerations become important, which are bridging intercalation and surface science.

\subsection{Staging in bulk materials}

As depicted in Fig. 2A surface functionalization differs from intercalation. The most important concept for describing intercalated materials is staging, which is used to describe the structure of layered materials with guest atoms, ions or molecules. This concept is best studied for GICs. Accordingly, stage $n$ was defined with $n$ as number of layers. Thus, if we define A as materials layer and $B$ as intercalant layer, stage 1 possesses an ABABAB sequence (Fig. 2B), stage 2 AABAABAAB sequence (Fig. 2C), stage 3 AAABAAAB sequence etc.

However, also mixed systems are possible with e.g. partiallyfilled layers, in particular occurring for natural systems, as a consequence of the kinetic control of intercalation (Fig. 2D). It is obvious that a small intercalant must enter the galleries of a layered bulk material from the side and intercalation starts from all rims at the same time. However, for the example of a stage 2 compound the hypothetical left rim intercalant does

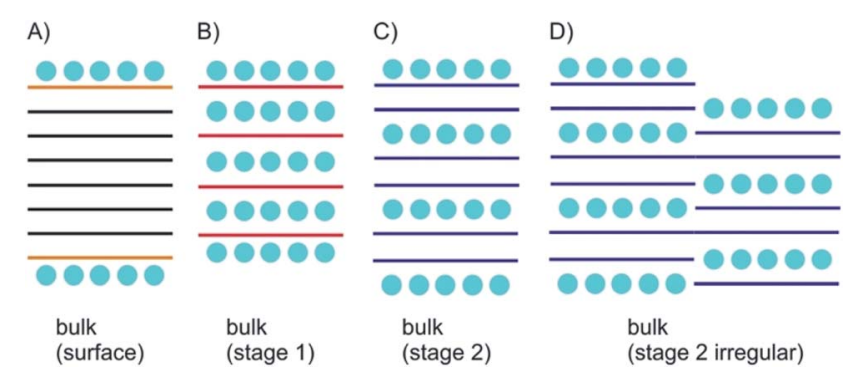

Fig. 2 Concept of bulk surface functionalization/intercalation. (A) Layered bulk material with surface functionalization; the interior is not influenced; (B) stage 1 intercalation compound; (C) stage 2 intercalation compound; (D) irregular, ill-defined stage 2 intercalation compound.

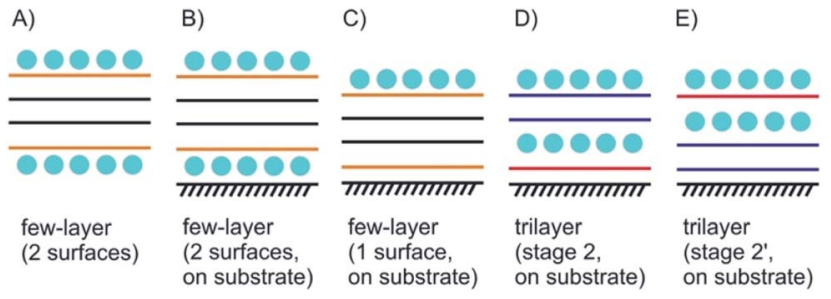

Fig. 3 Concept of few-layer surface functionalization/intercalation. (A) Surface functionalized few-layer material, here the interior $2 \mathrm{~L}$ differ from the outer layers; $(B)$ as in (A), but with substrate influence leading to Janus-type properties; (C) few-layered material with intercalation only on the top; (D) and (E) illustration of stage 2 and stage $2^{\prime}$ intercalation for $3 \mathrm{~L}$ materials.

not know at which layer the intercalant on the right rim starts. Such a mismatch cannot be corrected and will consequently lead to a mixed layer sequence. For a $2 \mathrm{~L}$ material there is only one gallery to be intercalated. With the given examples, the difference between surface manipulation, by interacting molecules or atoms, and intercalation becomes clear, since with intercalation layers, the bulk can be influenced from inside. With thinning the layered materials, the surface becomes more important for manipulating materials properties and thus, interesting effects emerge as outlined in the next chapters.

\subsection{From bulk to few-layers of intercalated 2D materials}

Few-layered 2DMs are obtained at first sight with thinning down from bulk. ${ }^{21}$ However, the concept of staging loses its validity with reaching $1 \mathrm{~L}$, instead surface science describes the systems. Here, we introduce some of the possible structures for few-layered systems, followed by three, two and one layers of 2DMs (Fig. 3 and 4).

As shown in Fig. 3A the four layers (4L) are an example of a few-layered material with the surface influenced by molecules or atoms. In that example with $4 \mathrm{~L}$ the orange marked layers can be distinguished from the inner black marked layers and there is consequently a junction between orange and black layers. However, for more than roughly six layers surface functionalization does not play a crucial role for the interior layers, since the proportion of surface layers to the interior layers becomes minor.

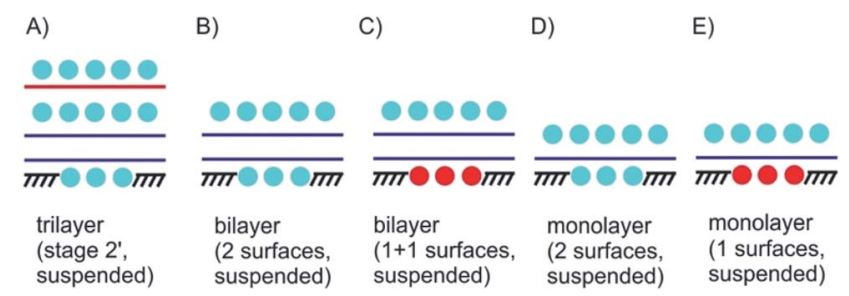

Fig. 4 Suspended $3 \mathrm{~L}, 2 \mathrm{~L}$ and $1 \mathrm{~L}$ materials with surface functionalization/intercalation. (A) $3 \mathrm{~L}$ stage $2^{\prime}$ intercalated and bottom surface functionalization; (B) surface functionalized suspended 2L; (C) Janustype surface functionalized $2 \mathrm{~L}$; (D) $1 \mathrm{~L}$ material intercalated between molecular layers; (E) Janus-type suspended $1 \mathrm{~L}$ material; $(D)$ and $(E)$ could be considered as 2D 1L intercalated between molecular layers. 
A)

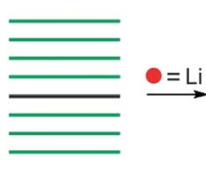

$\mathrm{hBN} / \mathrm{G} / \mathrm{hBN}$

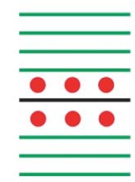

hBN/Li/G/Li/hBN
B)

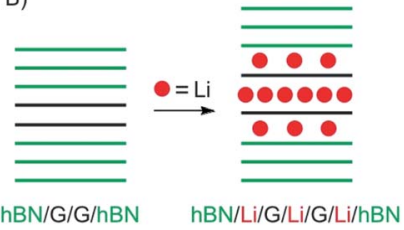

Fig. 5 Study of device-based electrochemical intercalation of Li in heterostructures of $(A)$ graphene $(G)$ artificially intercalated in hBN and (B) $2 \mathrm{~L}$ graphene artificially intercalated in $\mathrm{hBN}$.

Even for the example of 4 Ls the substrate plays an important role. As illustrated in Fig. 3B and $\mathrm{C}$, with considering a substrate, Janus-type functionalization (different functionalities on two sides) is realized no matter whether atoms or molecules are placed between the substrate and the 2DM or not. For the $3 \mathrm{~L}$ examples in Fig. 3D and $\mathrm{E}$ the concept of staging is adopted, here, with stage 2 as an example. Starting with a $3 \mathrm{~L}$ on a substrate and an intercalant preferring stage 2 formation, two different configurations can be considered, and thus, either a $1 \mathrm{~L}$ or $2 \mathrm{~L}$ is on the substrate. Here, we introduce stage 2 and stage $2^{\prime}$, respectively, to differentiate between those two cases. The example in Fig. 3D starts from the top like a stage 2 compound. Thus, we assign stage $2^{\prime}$ to the example in Fig. 3E.

In Fig. 4A a stage $2^{\prime}$ structure is shown, however, in contrast to Fig. $3 \mathrm{E}$ the structure is suspended, which can be realized by placing the structure on top of a hole in the substrate. A similar structure is shown in Fig. 1A for a $2 \mathrm{~L}$ of graphene. In general, with suspending few-layered 2DMs transmission is possible, however, also surface manipulation, such as doping becomes possible accounting for different surfaces. This is the point where surface science plays the decisive role in manipulating 2DMs. For a $2 \mathrm{~L}$ both surfaces can be accessed by molecules or atoms, which are the same (Fig. 4B) or different ones (Fig. 4C); the latter leading to Janus-type functionalization. Taking the concept of staging into account a stage 2 configuration can be realized for a $2 \mathrm{~L}$, although no intercalation occurs. For a $1 \mathrm{~L}$ similar surface functionalization is possible, as shown in Fig. 4D and E. In those examples, the $1 \mathrm{~L}$ is however intercalated between molecular layers and thus, surface science and intercalation chemistry merge.

In a recent study, intercalation of vdW heterostructures of graphene and 2L graphene, respectively, was studied in an electrochemical device (as outlined in chapter 3 ). ${ }^{22}$ The structures prepared are illustrated in Fig. 5A and B. As shown in Fig. 5A intercalation proceeds at the interface between hBN and graphene, on both sides. However, the properties differ from pure surface functionalization or a stage 1 bulk intercalation compound, due to the interaction of $\mathrm{Li}$ and hBN (about 30L on each side). The authors conclude that $\mathrm{Li}$ is randomly distributed, and the amount of $\mathrm{Li}$ atoms is significantly lower compared to the bulk with carrier densities of about $7 \times 10^{13}$ $\mathrm{cm}^{-2}$, corresponding to a stoichiometry of $\mathrm{LiC}_{60}$.

In contrast, a $2 \mathrm{~L}$ of graphene intercalated in $\mathrm{hBN}$, as shown in Fig. 5B, behaves differently because intercalation of the $2 \mathrm{~L}$ allows a much higher loading compared to the graphene/hBN

interface. Here, the carrier density was determined to $5.5 \times$ $10^{14} \mathrm{~cm}^{-2}$, with a contribution of $4.6 \times 10^{14} \mathrm{~cm}^{-2}$ for the graphene/graphene interface.

\subsection{Considerations on geometry and charge carrier densities}

When approaching a 1L of a $2 \mathrm{DM}$, or by stacking few-layers of 2DMs on top of each other or by intercalating materials, atomicscale illustrations become important to imagine the dimensions. In this regard, structures are often evaluated by transport measurements in devices. As a result, the mobility of charge carriers is determined, either in field effect transistors or in magnetic fields by Hall-bar measurements. Finally, taking the dimensions of the device into account a charge carrier density is derived with the unit $\mathrm{cm}^{-2}$ for 2DMs. However, for an atomic imagination the unit cell dimensions are important to take into account. For graphene, the unit cell contains two carbon atoms, with two equal lattice vectors. The calculated area for one carbon atom is $0.026195 \mathrm{~nm}^{2}$ or $0.026195 \times 10^{-14} \mathrm{~cm}^{2}$ (order of magnitude comparable to typical charge carrier densities), respectively. ${ }^{23}$ This means that there are $38 \times 10^{14} \mathrm{C}$-atoms per $\mathrm{cm}^{2}$. For the example illustrated in Fig. 5, this consideration means that there is one charge on about $14 \mathrm{C}$-atoms, taking the $2 \mathrm{~L}$ structure and interaction of all intercalated Li-atoms into account.

For $\mathrm{MoS}_{2}$ the area of the unit cell is $0.088 \mathrm{~nm}^{2}$ including one Mo and two S atoms, of which one points up and one down. Accordingly, there are $11 \times 10^{14}$ Mo-atoms per $\mathrm{cm}^{2}$ and $22 \times$ $10^{14} \mathrm{~S}$-atoms per $\mathrm{cm}^{2}$. In another example the intercalated heterostructure $\mathrm{MoS}_{2} / \mathrm{Li} /$ graphene was analyzed by density functional theory calculations,${ }^{24}$ with a charge carrier density of $3.6 \times 10^{14} \mathrm{~cm}^{-2}$ for graphene and $6.0 \times 10^{14} \mathrm{~cm}^{-2}$ for $\mathrm{MoS}_{2}$. According to the considerations above those value relate to one charge carrier on about $10.6 \mathrm{C}$-atoms and $3.7 \mathrm{~S}$-atoms, respectively.

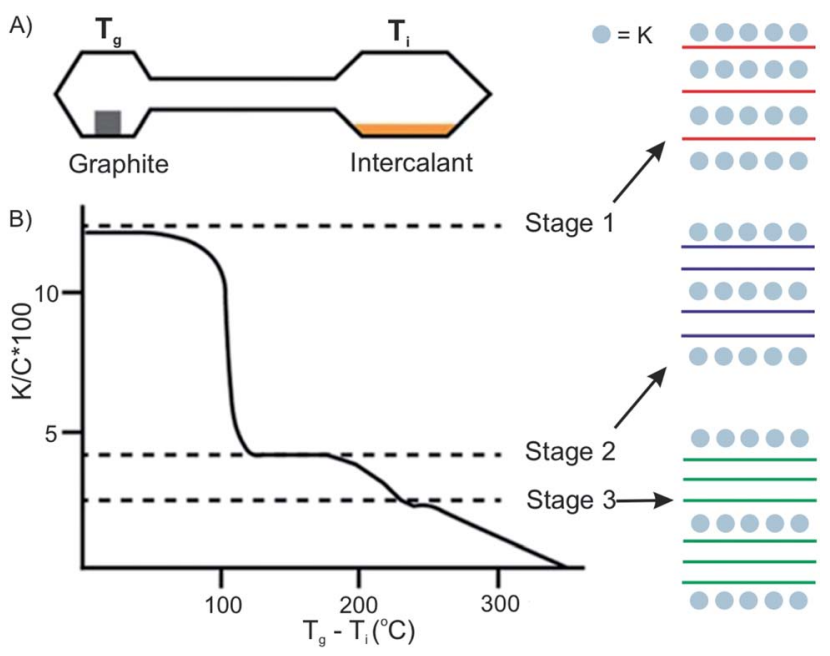

Fig. 6 Two-zone thermal transport for potassium into graphite and stage control by temperature difference. Reproduced from ref. 16 with permission from Wiley-VCH Verlag GmbH \& Co, Copyright 2019. 


\section{Synthetic access to intercalated 2D materials}

\subsection{Intercalation by vapor-transport}

The vapor transport method usually proceeds under vacuum or an inert gas atmosphere in a sealed two-zone cell (Fig. 6A). ${ }^{12}$ The intercalants and host materials are placed in separate zones, which are heated under different or same temperatures, respectively. The success of intercalation depends on controlling the temperature of the host zone and intercalant zone. Also, the stage of the intercalation compounds can be controlled by temperature adjustments. The temperature of the two zones can be different or isotherm, depending on the chemical and physical properties of the intercalant and the host material. ${ }^{25}$ For example, the stage of the prototype intercalation compound K-GICs is controlled by the temperature difference $T_{\mathrm{g}}-T_{\mathrm{i}}\left(T_{\mathrm{g}}\right.$ : temperature of graphite; $T_{\mathrm{i}}$ : temperature of intercalant) as shown in Fig. 6B. ${ }^{26}$ The higher stages are formed at a smaller $T_{\mathrm{g}}$ $-T_{\mathrm{i}}$.

In the case of halogen intercalation, typical preparation temperatures are around $20^{\circ} \mathrm{C}$ to $60^{\circ} \mathrm{C}$ for $T_{\mathrm{g}}$. Owing to the high threshold vapor pressure of the halogens, such as $\mathrm{Br}_{2},{ }^{27} \mathrm{IBr}$ and $\mathrm{ICl}, T_{\mathrm{i}}$ is set from $-30{ }^{\circ} \mathrm{C}$ to $60^{\circ} \mathrm{C}$ to control the pressure in the reaction cell. ${ }^{28}$ Other parameters, including heating time, the
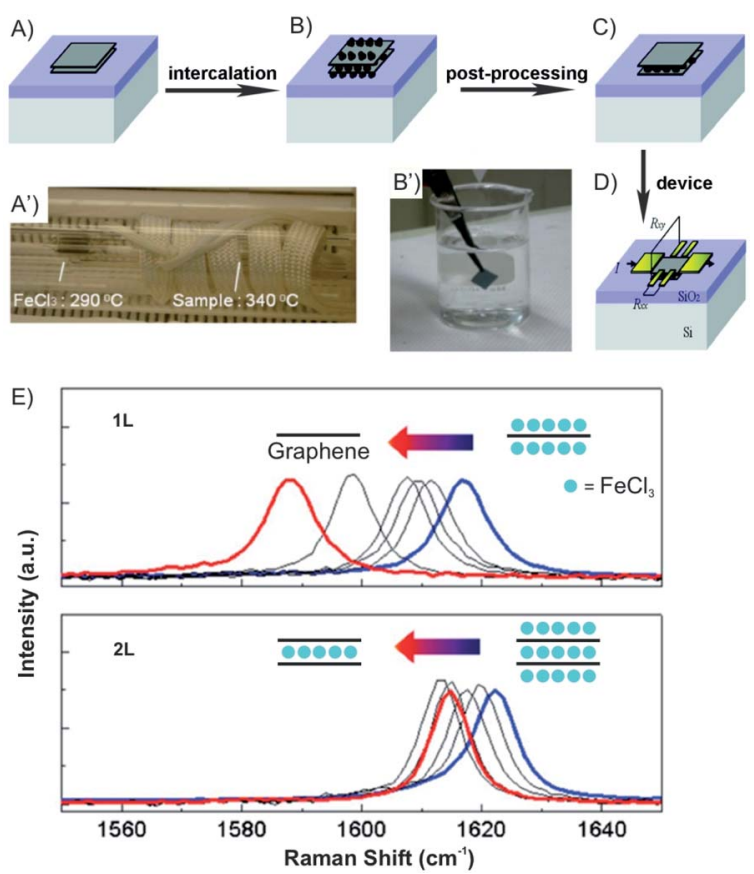

Fig. 7 Fabrication of an $\mathrm{FeCl}_{3}$ intercalated $2 \mathrm{~L}$ graphene device. (A) Pristine and (B) after $\mathrm{FeCl}_{3}$ intercalation of $2 \mathrm{~L}$ graphene deposited on $\mathrm{Si} / \mathrm{SiO}_{2}$ substrate. $\left(A^{\prime}\right)$ The sealed tube is placed in the furnace. $\left(\mathrm{B}^{\prime}\right)$ Sufficient washing with acetone $(C)$ removes the adsorbed molecules from the surface. (D) Deposition of electrodes using standard lithography fabrication techniques. (E) Gradual downshifts of the Raman G peaks is observed (after intercalation without air exposure; blue) and after washing in acetone for 0, 0.16, 0.5, 1 (black), and $12 \mathrm{~h}$ (red). Adapted from ref. 47 with permission from the American Chemical Society, Copyright 2011. size of host materials, the amount of intercalant, and the volume and shape of ampoule also affect the homogeneity and in general the quality of the final compound. ${ }^{12,29,30}$

Vapor transport intercalation is the most widely applied method for the intercalation of bulk materials, because it works for most of the possible hosts, such as graphite, ${ }^{\mathbf{1 2}}$ fullerenes, ${ }^{\mathbf{3 1}}$ single-walled carbon nanotubes, ${ }^{32} \mathrm{hBN}^{33}$ and TMDCs $\left(\mathrm{MoS}_{2}\right.$, $\mathrm{TaS}_{2}, \mathrm{WS}_{2}$ and $\left.\mathrm{WSe}_{2}\right)^{34-36}$ and a variety of intercalants, such as alkali metal $(\mathrm{K}, \mathrm{Rb} \text { and } \mathrm{Cs})^{26,37,38}$ or their alloys, ${ }^{39,40}$ halogens $\left(\mathrm{Br}_{2}\right)^{27}$ or interhalogens ( $\mathrm{IBr}$ and ICl), ${ }^{28,41}$ metal halides $\left(\mathrm{FeCl}_{3}\right.$, $\mathrm{AlCl}_{3}, \mathrm{SbCl}_{5}$ and $\left.\mathrm{AsF}_{5}\right)^{\mathbf{4 2 - 4 5}}$ and organic molecules (pyridine, cyclopropylamine). ${ }^{46}$ This method is already well developed for bulk intercalation. Thus, it can be easily and directly applied to $2 \mathrm{D}$ host materials using the same equipment (two-zone cell) and similar experimental conditions (temperature). The 2D host is normally deposited on a substrate or a manufactured device that can be directly inserted into the reaction cell, as depicted in Fig. 7A-D, illustrating $\mathrm{FeCl}_{3}$ intercalation. ${ }^{47}$ With reduced lateral dimensions, the intercalation rate of few-layered materials is much faster compared to the bulk. The synthesis of stage 1 alkali metal (K, Rb) GICs usually takes more than $12 \mathrm{~h},{ }^{\mathbf{4 8}}$ while only $5 \mathrm{~min}$ are needed for the intercalation of $1-15 \mathrm{~L}$ of graphene. ${ }^{49}$

Over the past decade, progress has been made in few-layered 2DMs intercalation by the vapor transport method. The 2DM intercalation compounds were studied and some showed similarities to their bulk intercalation compound, such as the formed stage, ${ }^{\mathbf{4 9 , 5 0}}$ which offers good references for the investigation and characterization of few-layered 2DMs intercalation.
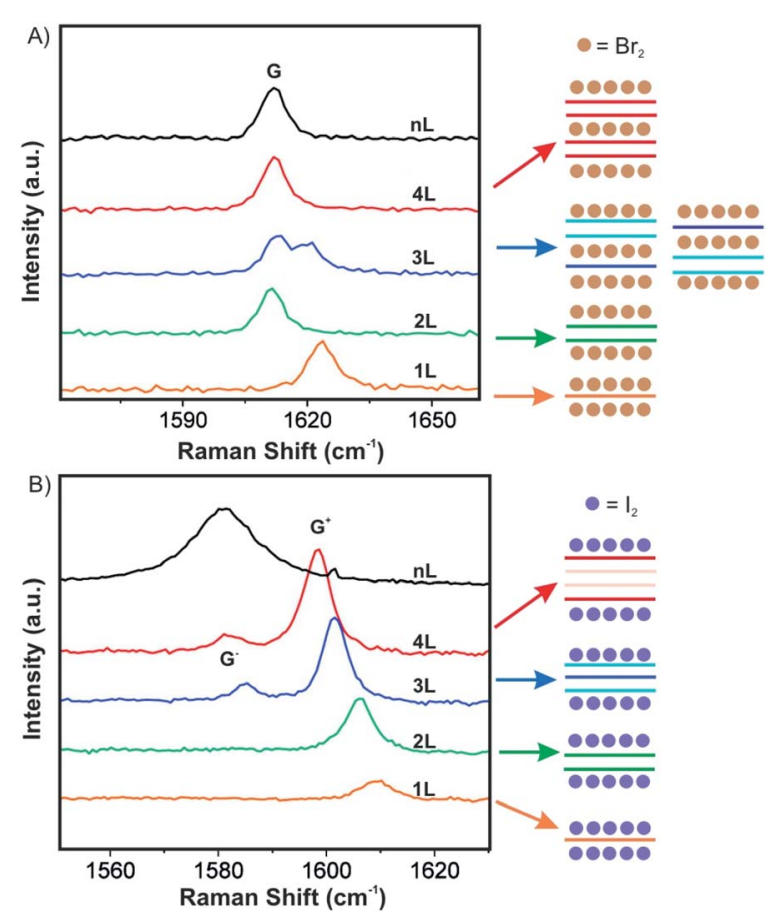

Fig. 8 Raman spectra of few layer graphene materials exposed to (A) $\mathrm{Br}_{2}$ and $(\mathrm{B}) \mathrm{I}_{2}$ and the derived intercalation models. Adapted from ref. 50 with permission from the American Chemical Society, Copyright 2009 
For few-layered 2DMs the effect of surface adsorption of intercalants becomes non-negligible as discussed in chapter 2. For example, $\mathrm{Br}_{2}$ and $\mathrm{I}_{2}$ have been attempted to intercalate in mechanically exfoliated $1-4 \mathrm{~L}$ of graphene on a p-type $\mathrm{Si} / \mathrm{SiO}_{2}$ wafer. ${ }^{50,51}$ The wafer with host materials was placed in one zone of a glass cell, while liquid halogen was placed in another zone at a temperature of $10{ }^{\circ} \mathrm{C}$ to keep a constant vapor pressure in the cell. To avoid any impurities, the tube cell was initially evacuated to $2.7 \times 10^{-5}$ mbar and the liquid halogen was frozen and thawed several times to remove dissolved gases. After $1 \mathrm{~h}$ of $\mathrm{Br}_{2}$ exposure, $\mathrm{Br}_{2}$ was successfully intercalated into $3 \mathrm{~L}$ and $4 \mathrm{~L}$ of graphene, respectively. In contrast, for $2 \mathrm{~L}$ and $1 \mathrm{~L}$ graphene, $\mathrm{Br}_{2}$ was symmetrically adsorbed on the top and bottom surfaces. Those results are consistent with the observations made for the $\mathrm{Br}_{2}$-GIC, with stage 2 as the lowest reported stage. ${ }^{27}$ Accordingly, the model as shown in Fig. 8A is concluded from Raman spectra of the intercalation compounds. A single $\mathrm{G}$ peak near $1612 \mathrm{~cm}^{-1}$ is found for $2 \mathrm{~L}$ and $4 \mathrm{~L}$ graphene, which are symmetrically intercalated and doped, similar to the bulk $\mathrm{Br}_{2}$-GIC reference. However, the $1 \mathrm{~L}$ showed an upshift of the G peak to $1614 \mathrm{~cm}^{-1}$, which relates to a higher charge carrier concentration for the single graphene layer. For intercalated $3 \mathrm{~L}$ graphene, two $\mathrm{G}$ peaks are detected, which indicates two different types of inequivalent graphene layers as a result of asymmetric intercalation.

Few-layer graphene was also exposed to $\mathrm{I}_{2}$. Raman spectra for $3 \mathrm{~L}$ and more layers treated with $\mathrm{I}_{2}$ showed two G peaks (Fig. 8B). This means $\mathrm{I}_{2}$ adsorbs only on the top and the bottom of fewlayer graphene without intercalation, possibly because the longer $\mathrm{I}_{2}$ bond length does not allow an intercalation structure. ${ }^{52}$ Similar to bulk intercalation, $\mathrm{Br}_{2}$ is also the only diatomic nonpolar halogen molecule that can be easily intercalated into graphite. However, iodine in interhalogen compounds such as $\mathrm{ICl}$ and $\mathrm{IBr}$ can be intercalated..$^{53}$

Compared to alkali metal intercalation compounds, it is reported that $\mathrm{FeCl}_{3}$ intercalation compounds are relatively stable at ambient conditions. ${ }^{54}$ Those materials can be safely removed

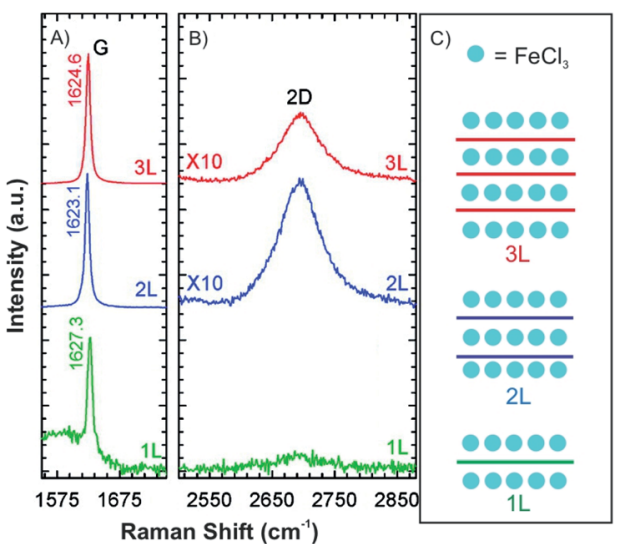

Fig. 9 (A) G peak, (B) 2D peak and (C) schematic illustrations of stage 1 for $1 \mathrm{~L}, 2 \mathrm{~L}$ and $3 \mathrm{~L}$ graphene- $\mathrm{FeCl}_{3}$ intercalation compounds. Adapted from ref. 56 with permission from the American Chemical Society, Copyright 2011. from their encapsulating ampoules for short periods of time, and therefore, provide prototype materials for the measurements to determine properties and further exploratory investigations. Due to that, $\mathrm{FeCl}_{3}$ intercalation was investigated on the 2D level. ${ }^{47,55-58}$ Accordingly, 1-4L graphene flakes were intercalated by $\mathrm{FeCl}_{3}$ to a stage 1 intercalation compound $\left(\mathrm{FeCl}_{3}\right.$-fewlayered graphene (FLGs), Fig. 9). ${ }^{56}$ Owing to the highly hygroscopic property of $\mathrm{FeCl}_{3}$, it was heated at $393 \mathrm{~K}$ for more than $90 \mathrm{~min}$ to remove any residual water. $\mathrm{Next}, \mathrm{FeCl}_{3}$ and the host were sealed in an ampoule and inserted in an oven at a reaction temperature of $613 \mathrm{~K}$ for $6 \mathrm{~h}$. The Raman spectra for $1 \mathrm{~L}, 2 \mathrm{~L}$ and $3 \mathrm{~L}$ graphene display an upshift of the $\mathrm{G}$ peak and a change of the $2 \mathrm{D}$ peak from a multi- to a single-peak structure. This is an indication of electronic decoupling of the layers by the intercalant for $2 \mathrm{~L}$ and $3 \mathrm{~L}$ graphene intercalated by $\mathrm{FeCl}_{3}$. The single $\mathrm{G}$ peak also indicates uniform adsorption and intercalation of layers by $\mathrm{FeCl}_{3}$ (Fig. 9A). For $1 \mathrm{~L}$ graphene both surfaces adsorb $\mathrm{FeCl}_{3}$ and the highest upshift of the $\mathrm{G}$ peak is observed at $1627 \mathrm{~cm}^{-1}$, compared to $1623 \mathrm{~cm}^{-1}$ for $\mathrm{FeCl}_{3}$ intercalated $2 \mathrm{~L}$ and $\mathrm{FeCl}_{3}$ intercalated $3 \mathrm{~L}$, respectively. However, multiple $\mathrm{G}$ peaks also have been reported in the Raman spectra of stage 1 $\mathrm{FeCl}_{3}$-FLGs. ${ }^{56-58}$ There are two explanations for this observation: (1) $\mathrm{FeCl}_{3}$ molecules only intercalate between interlayers without adsorbing on the surface layers of graphene. The lower $\mathrm{G}_{1}$ peak is a signature of a graphene sheet with only one adjacent $\mathrm{FeCl}_{3}$ layer, whereas the higher shift $\mathrm{G}_{2}$ peak characterizes a graphene sheet sandwiched between two $\mathrm{FeCl}_{3}$ layers. ${ }^{58}$ (2) The additional G peak is caused by nonuniform intercalation, due to desorption of $\mathrm{FeCl}_{3}$ during cooling down in vacuum. To prove the hypotheses, $\mathrm{FeCl}_{3}$-FLGs were exposed to air by two ways. In the first approach, $\mathrm{FeCl}_{3}$-FLGs were immediately removed from the hot glass tube. In the second approach, the glass tube was first air-cooled to room temperature while still sealed and then the samples were removed. The samples obtained from the first method exhibited a single $\mathrm{G}$ peak and a higher doping level, which was stable up to one month in air. ${ }^{56}$ Moreover, as shown

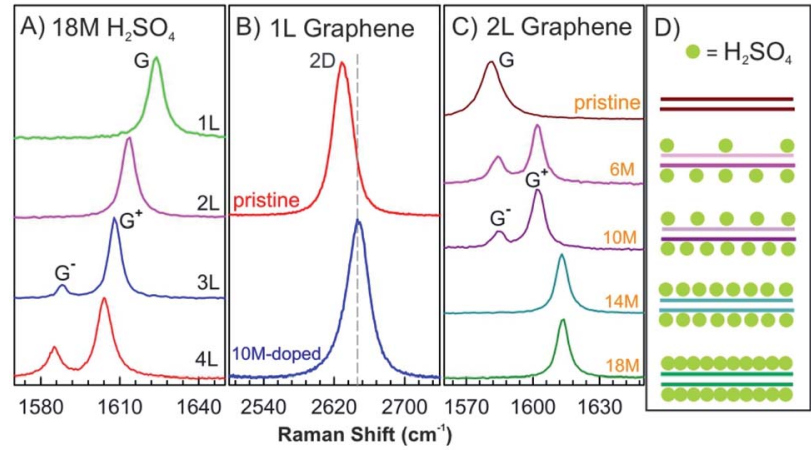

Fig. 10 (A) In situ Raman spectra of the G peak of 1-4L graphene samples dipped into $18 \mathrm{M}$ sulfuric acid. (B) The $2 \mathrm{D}$ peak of pristine $1 \mathrm{~L}$ graphene and that doped by $10 \mathrm{M}$ sulfuric acids. (C) In situ Raman spectra of the $G$ peak of the $2 L$ graphene doped by different sulfuric acid concentrations of $18 \mathrm{M}, 14 \mathrm{M}, 10 \mathrm{M}$, and $6 \mathrm{M}$ and a pristine BLG as a reference. (D) Structural model of $2 \mathrm{~L}$ graphene structures doped with sulfuric acid derived from Raman spectra in (C). Adapted from ref. 62 with permission from the American Physical Society, Copyright 2010. 


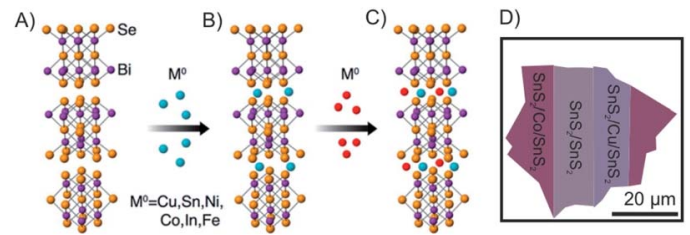

E)

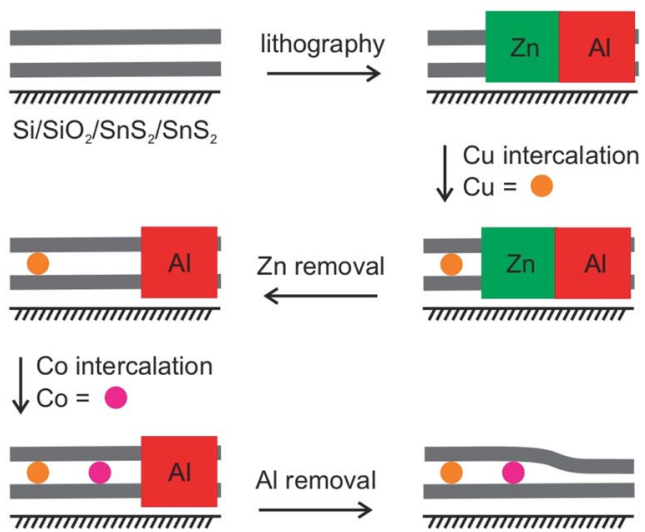

Fig. 11 Intercalation of (A and $\mathrm{B}$ ) single and multiple (C) zero-valent metal atoms into $\mathrm{Bi}_{2} \mathrm{Se}_{3}$. Adapted from ref. 66 with permission from the American Chemical Society, Copyright 2015. (D) Schematic illustration of seamlessly integrated n-type $\mathrm{SnS}_{2}, \mathrm{p}$-type $\mathrm{Cu}-\mathrm{SnS}_{2}$ and metallic $\mathrm{Co}-\mathrm{SnS}_{2}$ within a single nanosheet. (E) Schematics of the spatially controlled intercalation process for $2 \mathrm{~L} \mathrm{SnS} 2 .{ }^{67}$

in Fig. 7B and $\mathrm{C}$, adsorbed $\mathrm{FeCl}_{3}$ on graphene surface can be simply removed by washing in acetone. According to the Raman spectrum, the G peak shifts down continuously with increased washing time. Finally, a $1 \mathrm{~L}$ is recovered with a normal $\mathrm{G}$ peak position, which decreased to $\sim 1580 \mathrm{~cm}^{-1}$, while the G peak of $2 \mathrm{~L}$ graphene did not shift back to the undoped value (Fig. 7E). This difference indicates that the intercalated molecules are more stable owing to the protection between graphene layers, but would be eventually deintercalated by more extensive rinsing in acetone or other solvents such as hydrochloric acid. ${ }^{47}$

Through vapor transport intercalation, a lot of intercalant and host pairs have been fabricated on the bulk level (as mentioned above), but only few are reported for few-layered
2DMs. As the vapor transport method can directly be applied for few-layered 2DMs intercalation, more intercalants and host materials can be investigated in the future. However, the instability of the intercalation compounds hampers the realtime characterization and thus, also limits further investigations.

\subsection{Solvent-based intercalation}

Nonelectrochemical solvent-based intercalation is easy to apply, compared to other methods, since no special equipment or reaction conditions are necessary. Typically, the host material is soaked in a solution of the intercalant. The intercalation process is often accompanied by chemical reactions, enabling intercalation or post reactions. The most representative example is the synthesis of graphite oxide by Hummers method. ${ }^{59}$ In the first step, graphite is dispersed in sulfuric acid with an oxidant to form graphite sulfate, an intercalation compound with the stoichiometry $\left[\mathrm{C}_{24}{ }^{+} \mathrm{HSO}_{4}{ }^{-} \cdot 2 \mathrm{H}_{2} \mathrm{SO}_{4}\right]_{n}{ }^{10,60}$ During this process the oxidant p-dopes graphite enabling intercalation of hydrogensulfate counterions and sulfuric acid molecules. ${ }^{61}$ With adding more oxidant, such as potassium permanganate further chemical modifications occur on both sides of the interlayers and finally covalent $\mathrm{C}-\mathrm{O}$ bonds are formed. In this way, graphite oxide is formed, which can be delaminated to graphene oxide. ${ }^{3}$

Nevertheless, in the absence of oxidizing agents, this reaction works differently for 1-4L of graphene hosts, for which sulfuric acid molecules are found to be only physically adsorbed on the top and bottom layers without intercalation. ${ }^{62}$ The $\mathrm{G}$ peaks of $1-4 \mathrm{~L}$ of graphene in $18 \mathrm{M}$ sulfuric acid show similar Raman spectra as observed for graphene few-layers with adsorbed $\mathrm{I}_{2}$, although the chemical doping of $\mathrm{I}_{2}$ vapours on graphene is quite different (Fig. $8 \mathrm{~B}$ and $10 \mathrm{~A}$ ). The $2 \mathrm{D}$ peak of $1 \mathrm{~L}$ graphene upshifts by about $10 \mathrm{~cm}^{-1}$ after dipping into $10 \mathrm{M}$ sulfuric acid, as shown in Fig. 10B, which is a typical characteristic of hole doping in graphene layers. ${ }^{63}$ As shown in Fig. 10C and $\mathrm{D}$, the doping level of the top and bottom graphene layers can be asymmetric by adjusting the concentration of sulfuric acid to $6 \mathrm{M}$ and $10 \mathrm{M}$, respectively. At a low concentration of sulfuric acid, the molecules are not completely adsorbed on the

Table 1 Summary of reactions to generate zerovalent species, and precursor chemistry. Adapted with permission from ref. 64 from the American Chemical Society, Copyright $2012^{a}$

\begin{tabular}{|c|c|c|}
\hline Intercalant & Precursor(s) & Reaction \\
\hline Copper & Tetrakis (acetonitrile) copper(I) hexafluorophosphate & $\left.2 \mathrm{Cu}_{(\mathrm{aq})}\right)^{+} \rightarrow \mathrm{Cu}_{(\mathrm{aq})}{ }^{2+}+\mathrm{Cu}(0)$ \\
\hline Silver & $\begin{array}{l}\text { Silver nitrate; } 0.1 \text { g } 5,5,7,12,12,14 \text {-hexamethyl-1,4,8,11- } \\
\text { tetraazocyclotetradecane }\end{array}$ & $2 \mathrm{Ag}^{+}+\mathrm{L} \rightarrow \mathrm{AgL}^{2+}+\mathrm{Ag}(0)$ \\
\hline Tin & Stannous chloride; $0.1 \mathrm{~g}$ tartaric acid & $2 \mathrm{Sn}^{2+} \rightarrow \mathrm{Sn}^{4+}+\mathrm{Sn}(0)$ \\
\hline Gold & Gold(I) chloride or chlorotristriphenylphosphine gold(I) & $3 \mathrm{Au}_{(\mathrm{aq})}^{+} \rightarrow \mathrm{Au}_{(\mathrm{aq})}{ }^{3+}+2 \mathrm{Au}(0)$ \\
\hline Indium & Indium(I) chloride & $3 \mathrm{InCl} \leftrightarrow \mathrm{InCl}_{3}+2 \operatorname{In}(0)$ \\
\hline Nickel & Nickel(II) nitrate pentahydrate; hydrazine hydrate & $2 \mathrm{Ni}^{2+}+\mathrm{N}_{2} \mathrm{H}_{4}+4 \mathrm{OH}^{-} \rightarrow 2 \mathrm{Ni}(0)+\mathrm{N}_{2}+4 \mathrm{H}_{2} \mathrm{O}$ \\
\hline
\end{tabular}

${ }^{a}{ }^{1} \mathrm{~L}=$ tetraazocyclic amine ligand. 
two surface layers, which causes the splitting of the G peak, indicating two graphene layers of different doping degree.

Another advantage of the solvent-based intercalation is that zero-valent metal intercalation in $2 \mathrm{D}$ host materials can be realized, which is difficult to achieve with other intercalation methods. As illustrated in Fig. 11A a variety of zero-valent metal atoms, including $\mathrm{Ag}, \mathrm{Au}, \mathrm{Co}, \mathrm{Cu}, \mathrm{Fe}, \mathrm{In}, \mathrm{Ni}$, and $\mathrm{Sn}$, were intercalated into $2 \mathrm{D}$ layered $\mathrm{Bi}_{2} \mathrm{Se}_{3}$ nanoribbons (50 nm thick). ${ }^{64}$ The zerovalent guest species were generated by a disproportionation redox reaction in solution or by carbonyl decomposition (Table 1) in a refluxing solution, and then intercalated into the layered $\mathrm{Bi}_{2} \mathrm{Se}_{3}$ structure. The atom\% of intercalant is controlled by either the concentration or the reaction time. Among all the metal atoms, $\mathrm{Cu}$ showed the highest intercalation concentration up to 60 atom\%, which is much larger than concentrations of compounds synthesized by heating or electrochemical insertion (below $\sim 3$ atom\%). ${ }^{65}$ The $\mathrm{Cu}$-intercalated nanoribbons appeared reddish, close to the colour of $\mathrm{Cu}$ metal, suggesting high concentrations of zero-valent copper. Additionally, 30 different ordered multiple pairs of zero-valent atomic species $(\mathrm{Cu}, \mathrm{Sn}, \mathrm{Ni}, \mathrm{Co}$, In and $\mathrm{Fe}$ ) were also intercalated into $\mathrm{Bi}_{2} \mathrm{Se}_{3}$ nanoribbons by a stepwise combination of different solvent-based intercalation processes to form $2 \mathrm{D}$ alloys inside the host lattice (Fig. 11A and B). Depending on the type of intercalant and their intercalating order, the intercalant exhibits a variety of ordered and disordered structures (including superlattices and charge density waves). In most cases, the intercalant remains at zero valence. ${ }^{66}$ Furthermore, chemical vapor deposition (CVD) grown n-type semiconducting 2L $\mathrm{SnS}_{2}$, which was intercalated with $\mathrm{Cu}$ and $\mathrm{Co}$ atoms, is transformed into a p-type semiconductor and a highly conductive metal. ${ }^{67}$ Combining this method with lithography, spatially controlled intercalation can be realized to seamlessly integrate n-type and p-type semiconductors and metals in 2DMs, which is difficult to achieve with mechanical transfer or other methods (Fig. 11D and E). ${ }^{67}$ These methods have been further extended to intercalate various TMDCs $\left(\mathrm{MoS}_{2}, \mathrm{MoSe}_{2}\right.$, $\left.\mathrm{NbSe}_{2}, \mathrm{WS}_{2}, \mathrm{Sb}_{2} \mathrm{Te}_{3}, \mathrm{In}_{2} \mathrm{Se}_{3}, \mathrm{GaSe}\right)^{68,69}$ and non-TMDs, such as $\mathrm{MoO}_{3},{ }^{70}$ as well as incorporating dual metal elements into those materials. As apparent from the reported results it seems that the solvent-based method to intercalate zero-valent metals in 2DMs is universal, although more investigations are necessary.

Solution-phase intercalation has also been applied to MXenes, ${ }^{71-73}$ a new family of $2 \mathrm{D}$ layered materials discovered in $2011 .^{74}$ Organic molecules $^{72,73}$ and alkali metal ions ${ }^{71,75}$ have been investigated for the intercalation of solid MXene nanosheets. For example, hydrazine monohydrate, hydrazine monohydrate dissolved in $N, N$-dimethylformamide (DMF), urea and dimethyl sulfoxide (DMSO) were successfully intercalated into hydrofluoric acid (HF) modified 2D MXene f- $\mathrm{Ti}_{3} \mathrm{C}_{2}\left(\mathrm{Ti}_{3} \mathrm{C}_{2}(-\right.$ $\left.\mathrm{OH})_{x} \mathrm{O}_{y} \mathrm{~F}_{z}\right) \cdot{ }^{72}$ To prove the universality of this method rather than the exclusive property of $\mathrm{f}-\mathrm{Ti}_{3} \mathrm{C}_{2}$, other MXenes $\mathrm{Ti}_{3} \mathrm{CN}$ and TiNbC were also intercalated by hydrazine monohydrate. The intercalation process can be reversed by heating the intercalated material above the boiling point of the intercalated species leading to a recovery of the c-lattice parameter. DMSOintercalated $\mathrm{f}-\mathrm{Ti}_{3} \mathrm{C}_{2}$ can be exfoliated due to its hygroscopic character by sonication in water affording a stable colloidal solution of separate sheets, from which a $\mathrm{Ti}_{3} \mathrm{C}_{2}$ film can be prepared on an $\mathrm{Al}$ membrane. Although there are many studies on bulk MXenes intercalation, ${ }^{75-78}$ investigations conducted on few-layered MXenes remains a field of research which is in progress.

Solvent-based intercalation can be applied easily to fewlayered 2DMs by immersing the substrate with the $2 \mathrm{D}$ host adsorbed on the surface into the solution of the intercalant. ${ }^{62,79}$ Unlike electrochemical intercalation, solvent-based intercalation can intercalate insulating host materials, such as hBN. ${ }^{\mathbf{8 0}}$ The concentration of intercalants is adjustable by the concentration of solutions, however precise control is hard to achieve. It is reported that these intercalation compounds are more stable than those formed by the vapor-transport method, and can thus be easily characterized. ${ }^{71,75}$ In addition, the intercalation may lead to exfoliation of the layered material or is reversible. These properties can be exploited either for preparing few-layered 2DMs, 2D intercalated materials or exploited in applications.

\subsection{Electrochemical intercalation}

Electrochemical intercalation allows to reversibly intercalate cations or anions into a layered host material driven by an external bias. For bulk intercalation, this is typically carried out in a three-electrode setup as depicted in Fig. 12. The host material serves as the working electrode (WE); therefore, it must be electrically conductive. The counter electrode (CE) is typically made of an inert metal plate or wire, e.g. Pt. A reference electrode (RE) provides a known potential (e.g. $\mathrm{Ag} / \mathrm{AgCl}$ or calomel) that is used to reference the relative potentials of the WE and CE during the experiment. The electrodes are placed in an aqueous, non-aqueous or solid-state electrolyte containing a salt for ion conductivity and the intercalant.

A direct or alternating current between the WE and CE is applied by an external power supply. The applied voltage is measured versus the potential between the RE and the WE. The intercalant enters the galleries of the host either via the vdW gaps or through defects of the top layer. ${ }^{82}$ The intercalation process can be followed in situ by the response of the current to the applied voltage. By further increasing the voltage, the

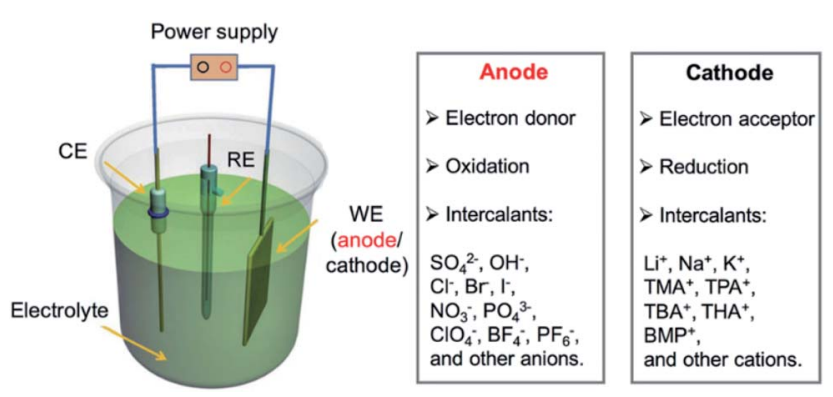

Fig. 12 Configuration of an electrochemical cell for bulk intercalation and typical reactions as well as typical intercalants. Reproduced from ref. 81 with permission from Wiley-VCH Verlag GmbH \& Co, Copyright 2020. 

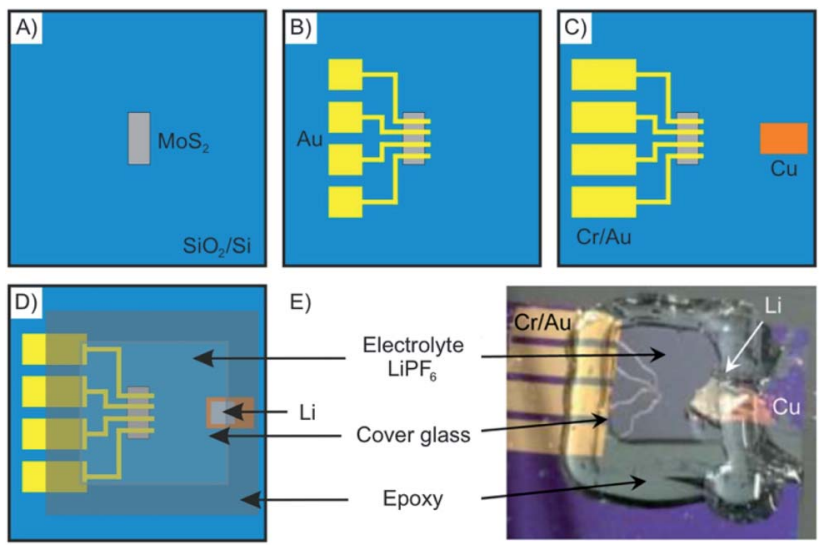

Fig. 13 Schematics showing the fabrication steps of an electrochemical device for in situ monitoring of $\mathrm{Li}$ intercalation into nanosheets of $\mathrm{MoS}_{2}$. (A) Mechanically exfoliated $\mathrm{MoS}_{2}$ flakes are deposited onto a $\mathrm{Si} / \mathrm{SiO}_{2}$-wafer. (B and $\mathrm{C}$ ) Metal electrodes are deposited via ebeam lithography and shadow mask evaporation. (D) The electrolyte is applied on top of the electrodes and a cover glass is used to sandwich the device that is sealed by epoxy resin to avoid oxidation. (E) Photograph of the as-prepared electrochemical intercalation device. Adapted from ref. 84 with permission from the American Chemical Society, Copyright 2015.

electrochemical intercalation can be used to functionalize or exfoliate few-layered 2DMs from a bulk sample. In aqueous solutions, e.g. GICs can be further oxidized to graphite oxide, which is subsequently delaminated to yield graphene oxide. ${ }^{\mathbf{8 3}}$ This approach is not limited to graphite but can be applied to a wide variety of 2DMs such as black phosphorous, TMDCs and transition metal carbides, as recently reviewed elsewhere. ${ }^{\mathbf{8 1}}$

Moving from the bulk to few-layered materials, the size of the experimental setup decreases accordingly. Microfabrication techniques are therefore required to fabricate electrochemical devices from few-layered 2DMs. The electrodes must be connected to a voltage source meter, either by placing on a conductive substrate or by contacting the flakes directly from the top by metal electrodes deposited onto the 2DM. In both cases the sample can lose electrical contact during the experiment due to swelling of the sample or deformation of the electrodes. The disadvantageous effect of swelling on the electrode contact may depend on the 2DMs thickness and should therefore be the smallest for a $2 \mathrm{~L}$ device.

As an example, in Fig. 13 the fabrication of a microscopic electrochemical intercalation device is depicted. The device enabled investigating the process of intercalation of Li into 2$50 \mathrm{~nm}$ thin $\mathrm{MoS}_{2}$. Such a typical device is fabricated starting by mechanical exfoliation of the 2DM. It is then dry-transferred onto a suitable substrate such as a $\mathrm{Si} / \mathrm{SiO}_{2}$ wafer (Fig. 13A). The 2DM can be subsequently etched into any desired shape to allow for example the in situ measurement of the Hall effect. The metal electrodes are then deposited by electron-beam lithography and physical vapor deposition (Fig. 13B and C). In the last fabrication step, the electrolyte solution containing the intercalant is applied onto the device (Fig. 13D and E). Measuring the small currents of the micrometer sized devices is challenging, however, possible.
In the case of few-layer intercalation of black phosphorous, the measured currents are in the range of tens of nanoampere. ${ }^{85}$ Zhao et al. encapsulated single and few-layers of graphene between hBN layers for shielding from the environment and to avoid parasitic currents originating from reactions in the electrolyte. ${ }^{22}$ The gold contacts used to contact the graphene layer were sealed using SU-8 resist to suppress any corrosion reactions at higher voltages.

The electrochemical intercalation between vdW heterostructures of different 2DMs is a huge new field of intercalation research. Zhao et al. not only used hBN to encapsule graphene layers and to avoid side reactions, but also studied the intercalation of $\mathrm{Li}$ ions at the interface of a single layer of graphene covered on both sides by hBN. ${ }^{22}$ Other vdW heterostructures of graphene and $\mathrm{MoS}_{2}$ were studied by Oakes et al. showing a large influence of strain at the interface of the two materials due to the lattice mismatch on the intercalation process. ${ }^{86}$ In another study Bediako et al. investigated Li intercalation at the interface of heterostructures of hBN, graphene, $\mathrm{MoS}_{2}$ and $\mathrm{MoSe}_{2} \cdot{ }^{87}$ This growing new field of research is also reviewed elsewhere. ${ }^{\mathbf{8 8}}$

Despite the possibility to electrochemical intercalation of a wide range of both anions, such as sulfates, ${ }^{89}$ nitrates $^{\mathbf{9 0}}$ and hexafluorophosphates ${ }^{91}$ and cations into bulk materials, most of the recent reports on the intercalation of few-layered materials are focused on the intercalation by alkali metals, while examples of anion intercalation are scarce. ${ }^{89}$ The intercalation of Li is by far the most studied process, which is motivated by the impact of its large-scale use in Li-ion battery technology. The intercalation of Li into few-layered 2DMs may help to better understand the underlying processes and guide further improvements of the technology. ${ }^{\mathbf{8 4 , 9 2 , 9 3}}$ As shown in the introductory example (Fig. 1), Kühne et al. showed superdense ordering of $\mathrm{Li}$ in $2 \mathrm{~L}$ graphene. ${ }^{15}$ They conclude that a $2 \mathrm{~L}$ of graphene may spread more easily upon intercalation compared to its bulk counterpart, showcasing the differences between fewlayer and bulk materials. In another study, they further showed very fast $\mathrm{Li}$ diffusion into $2 \mathrm{~L}$ graphene with a diffusion coefficient as high as $7 \times 10^{-5} \mathrm{~cm}^{2} \mathrm{~s}^{-1} \cdot{ }^{93}$ Aside from energy storage applications, electrochemical intercalation of charged organic

A)

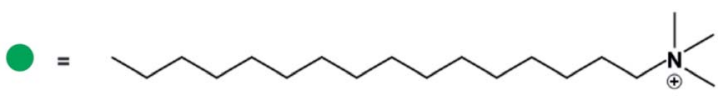

B)

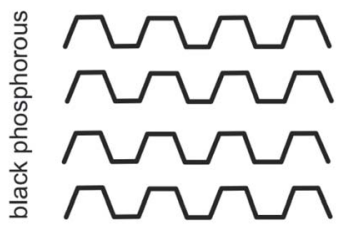

C)

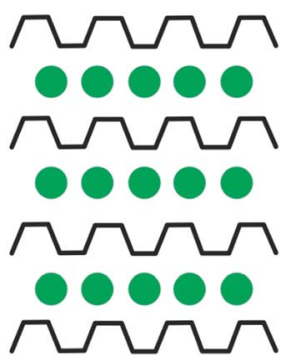

Fig. 14 (A) Illustration of the chemical structure of the cetyltrimethylammonium cation. (B) Schematic illustration of black phosphorous. (C) Schematic illustration of the stage 1 intercalation compound. ${ }^{85}$ 
molecules is used to fabricate well defined organic/vdW-hybrid heterostructures. ${ }^{\mathbf{8 5}, 94}$ By applying a potential of $-3 \mathrm{~V}$, Wang et al. intercalated multi-layered black phosphorous nanosheets with cetyltrimethylammonium cations (Fig. 14A). The intercalation could be followed by the response of the electrochemical gate current measured versus the applied voltage. The interlayer distance increased from $5.24 \AA$ to $11.21 \AA$ as illustrated in Fig. 14B and C. Thereby, superlattices of alternating molecule and 2DM layers were formed in which the individual phosphorene layers are electronically decoupled from each other. The decoupling of the phosphorene layers leads to an increased optical bandgap, as observed by in situ photoluminescence measurements. ${ }^{85}$

Recently, He et al. performed the electrochemical intercalation of the neutral organic semiconductor molecule perylene3,4,9,10-tetracarboxylic dianhydride (PTCDA) into few-layered $\mathrm{MoS}_{2}$ demonstrating a powerful design scheme for the fabrication of well-defined organic devices. ${ }^{95}$ This may bridge the gap from the research of 2DMs and vdW heterostructures and organic semiconductor research. Electrochemical intercalation of 2DMs enables a high control over the intercalation process by controlling the applied external bias. However, the 2DM must be conductive, excluding insulating materials such as hBN. Moreover, electrochemical intercalation requires the fabrication of microscale devices which increases the techniques complexity.

\subsection{Artificial assembly}

Artificial assembly, as sketched in the introduction, provides the opportunity to access distinct heterostructures to previously introduced conventional intercalation methods. The structures to be fabricated are not limited to certain ions or small molecules, which tend to intercalate specific hosts. Thus, the artificial assembly serves chances to integrate novel building blocks for the fabrication of few-layered intercalation compounds. However, the increasing freedom of parameters leads to complexity and thus a process, which is less controllable. For example, the integrity of the lattice of 1Ls may be harmed

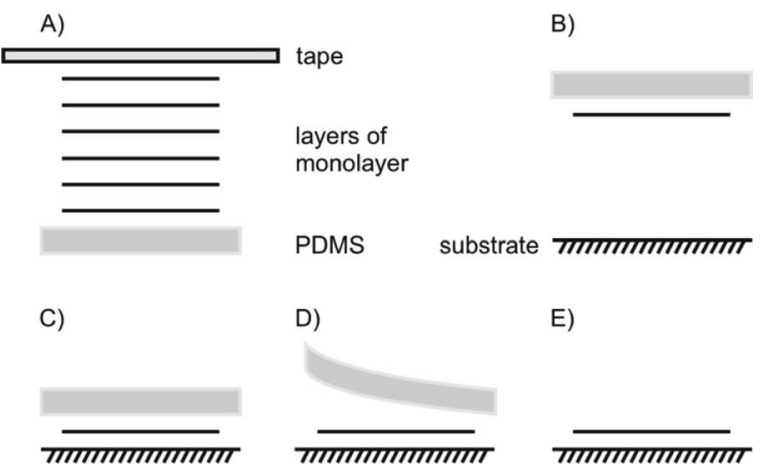

Fig. 15 Dry transfer with viscoelastic stamp of PDMS. (A) Tape exfoliation of bulk 2DM, (B) exfoliated $1 \mathrm{~L}$ on PDMS, upside down attached to a glass slide, that PDMS and the target substrate can be aligned by a micromanipulator, (C) contacting PDMS/1L and substrate, (D) lift off of PDMS leaving the $1 \mathrm{~L}$ on the substrate due to their stronger interaction, (E) transferred $1 \mathrm{~L}$ on new substrate. during exfoliation and transfer. Thereby cracks or folds may be formed. Furthermore, each process may introduce contaminants. Moreover, controlling the respective orientations in stacked lattices, twisted layers can be formed in which however unique properties emerge from lattice mismatch. ${ }^{\mathbf{9 6}-98}$ Furthermore, the orientation of ions/molecules or the number of layers are other degrees of freedom to be considered. However, with overcoming those issues, extraordinary structures with outstanding properties are created, as outlined in chapter 4 .

The mechanical transfer of 2DMs is based on overcoming vdW forces of stacked layers. It is a method to design structures of twisted layers, ${ }^{97-99}$ containing molecules, ${ }^{100,101}$ or of various 2DMs, such as graphene, hBN, TMDCs, black phosphorous or silicene. The yielded structures are also termed as heterostructures or vdW heterostructures. ${ }^{\text {102-104 }}$

The most common transfer method for flakes is a dry stamp technique using polydimethylsiloxane (PDMS) as illustrated in Fig. 15. It is an easily handled, clean, fast and reliable technique compared to others, like vdW pick up, usage of a sacrificial layer or wedging method. Those methods have been extensively compared by Frisenda et al. ${ }^{\mathbf{1 0 5}}$

With a PDMS stamp a 1L of bulk 2DMs can also be delaminated, a process, which is similar to the first invented scotch tape method (Fig. 15A). For the transfer, the flake is picked-up by the PDMS stamp, which is attached to a glass slide. The glass slide is fixed to a micromanipulator. Due to the transparency of PDMS, the alignment to an underlying flake or substrate can be traced under an optical microscope (Fig. 15B). After bringing PDMS and substrate in contact, PDMS can be slowly peeled off leaving the flake behind on the substrate (Fig. 15C-E).

The means of choice for large flake sizes, as can be produced by CVD, is a polymethylmethacrylate (PMMA)-supported etch transfer method (Fig. 16). ${ }^{106}$ Therefore, PMMA is dropped on a large $1 \mathrm{~L}$, spin casted and dried to stabilize the intact lattice (Fig. 16B). In the case of CVDgrown graphene the underlying substrate is a $\mathrm{Cu}$ foil, which is e.g. etched by a $\mathrm{Fe}\left(\mathrm{NO}_{3}\right)_{3}$ solution and washed with water. For a $\mathrm{Si} / \mathrm{SiO}_{2}$ substrate the removal of
A)

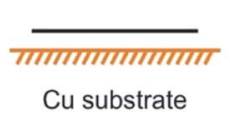

PMMA

monolayer

Si substrate
B)

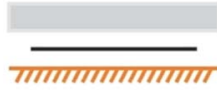

D)

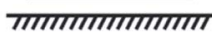

C)

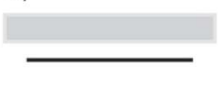

E)
Fig. 16 PMMA supported etching transfer commonly used for large areas of CVD grown materials. (A) $1 \mathrm{~L}$ on CVD supporting substrate (e.g. copper for CVD graphene). (B) Spin casting of PMMA on top of the $1 \mathrm{~L}$. (C) Removal of the substrate by etching. (D) Wet or dry transfer onto new substrate; wet transfer: after etching, PMMA/1L is washed in a water bath. By decreasing the water level, the 2DM can be transferred on a new substrate, which is placed at the bottom of the bath; dry transfer: an additional layer of PDMS is fixed on PMMA to handle $P M M A / 1 L$ without the stabilizing interface of water. (E) PMMA is removed by acetone leaving the CVD $1 \mathrm{~L}$ on new substrate. 
the substrate can be achieved by intercalation of water between the hydrophobic polymer/flake and hydrophilic substrate (named wedging transfer method; Fig. 16C). In both cases a subsequent wet-transfer may be performed by decreasing the water level. However, also a dry transfer is possible (Fig. 16D). ${ }^{107}$ Finally, PMMA is dissolved in acetone, rinsed and dried, leaving an intact lattice on the desired substrate (Fig. 16E).

Further methods are reported, ${ }^{\mathbf{1 0 5}, \mathbf{1 0 8}-\mathbf{1 1 1}}$ which are specific for materials, such as oxo-functionalized graphene, ${ }^{112}$ TMDCs $^{113}$ or requirements due to the shape of the substrate. ${ }^{\mathbf{1 1 4}}$

\section{Directions of research of intercalated few-layer 2D materials}

With thinning down the $z$-direction of layered 2DMs, properties changes, as introduced in the introduction. In this section, we highlight some research directions reported for intercalated few-layered 2DMs. In particular, we will focus on superconductivity, band-gap tuning, magnetic properties, optical properties, energy storage and chemical reactions.

\subsection{Superconductivity}

Superconductivity is a general research direction to find materials, which transport charge carriers at room temperature without resistance. The search for new superconductive materials is ongoing. In 1987 the Nobel prize in Physics was awarded to J. Georg Bednorz and K. Alex Müller "for their important break-through in the discovery of superconductivity in ceramic materials". ${ }^{115}$ Thereby, the research is based on the preparation of oxo-cuprates. Currently, $\mathrm{Hg}_{12} \mathrm{Tl}_{3} \mathrm{Ba}_{30} \mathrm{Ca}_{30} \mathrm{Cu}_{45} \mathrm{O}_{127}$ holds the world record for superconductivity at ambient pressure at 138 K. ${ }^{\mathbf{1 1 6}}$ Superhydrides, which possess structures of solid atomic metallic hydrogen, such as $\mathrm{LaH}_{10}$ (ref. 117) show a transition temperature of astonishing $259 \mathrm{~K}$, however, at $170 \mathrm{GPa} .{ }^{\mathbf{1 1 8}}$ The current world record holding superconductive materials are brittle and making km-long wires is not in reach. Thus, superconductivity in few-layered 2DMs would be the next superlative. Superconductivity is well-known for carbon materials, such as fullerenes, ${ }^{119}$ carbon nanotubes ${ }^{\mathbf{1 2 0}}$ and diamond. ${ }^{121}$ For graphene and doped graphene, superconductivity was predicted, ${ }^{\mathbf{1 2 1}}$ and for bulk $\mathrm{CaC}_{6}$, synthesized from graphite and $\mathrm{Li}-\mathrm{Ca}$ alloy, superconductivity was reported below $11.5 \mathrm{~K}^{122}$ A surprising discovery in this field was reported in 2018 for twisted $2 \mathrm{~L}$

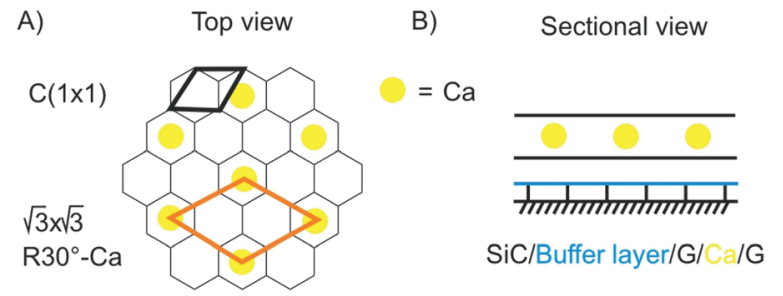

Fig. 17 Illustration of the structure of superconductive Ca-intercalated $2 \mathrm{~L}$ graphene. (A) Top view with graphene lattice in grey and position of $\mathrm{Ca}$ atoms in yellow. (B) Sectional view illustrating the layered structure. ${ }^{124}$ graphene with a twisting angle of $1.1^{\circ}$. At this "magic-angle" superconductivity is occurring at $1.7 \mathrm{~K} \cdot{ }^{97,123}$ In $2016 \mathrm{Ca}$ intercalated $2 \mathrm{~L}$ graphene $\left(\mathrm{C}_{6} \mathrm{CaC}_{6}\right)$ was reported to be superconductive at $4 \mathrm{~K}^{\mathbf{1 2 4}}$ The structure was prepared from epitaxial $2 \mathrm{~L}$ graphene. First Li atoms were intercalated and then exchanged by $\mathrm{Ca}$ (Fig. 17). Although this temperature is lower compared to the bulk, the experiment demonstrates that superconductive properties remain in $2 \mathrm{~L}$. Also other intercalated 2DMs are predicted to be superconductive, such as $2 \mathrm{~L}$ borophene $\left(\mathrm{B}_{2} \mathrm{MgB}_{2}\right)$ below 23 K. ${ }^{125}$

\subsection{Band gap tuning}

In this section, we highlight the possibilities to tune the electronic properties of $2 \mathrm{~L}$ and $3 \mathrm{~L}$ graphene by intercalation. Since graphene lacks a band gap, conventional transistors with $I_{\text {on }} / I_{\text {off }}$ ratios $>10^{6}$ are by far not possible..$^{\mathbf{1 2 , 1 2 6}}$ Thus, opening a band gap is necessary to generate a transistor behaviour with reasonable $I_{\text {on }} / I_{\text {off }}$ ratios. Breaking the inversion symmetry in $2 \mathrm{~L}$ graphene is one way to induce a band gap. ${ }^{127}$ It was reported that dual gating of $2 \mathrm{~L}$ graphene can open a band gap, reaching even an insulating state. ${ }^{128}$ With tuning the voltages of the top and bottom gate, tuning of the band gap of $2 \mathrm{~L}$ graphene was demonstrated with values up to $250 \mathrm{eV} \cdot{ }^{\mathbf{1 2 9}}$ Instead of dual-gating also intercalation/surface chemistry can induce a band gap.

Using density functional theory with vdW density functional, it was predicted that $2 \mathrm{~L}$ graphene can be intercalated between $\mathrm{K}$ on the bottom and $\mathrm{FeCl}_{3}$ on top to open a band gap in $2 \mathrm{~L}$ graphene with application relevant $0.4 \mathrm{eV}$ coming into reach. ${ }^{132}$ Experimentally, a $2 \mathrm{~L}$ of graphene was grown on $\mathrm{Ru}$ and

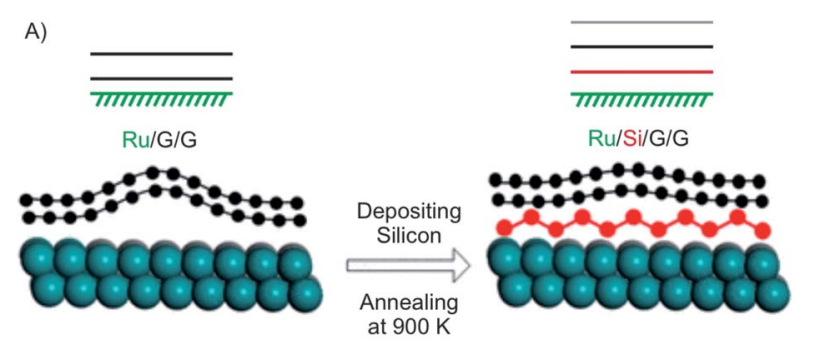

B)

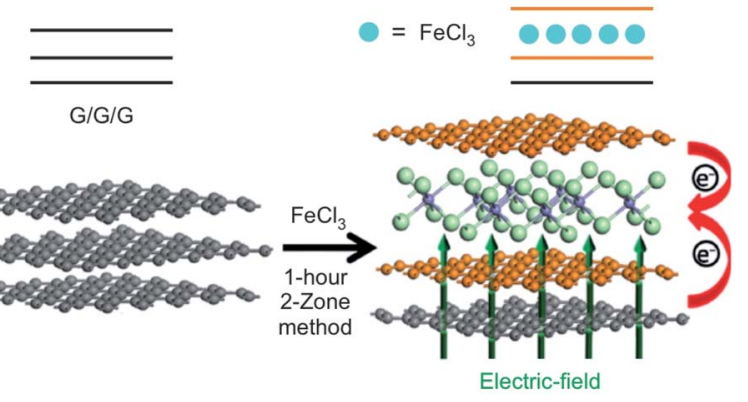

Fig. 18 (A) Illustration of the formation of the structure of Ru/Si/G/G with silicene intercalated between $\mathrm{Ru}$ and $2 \mathrm{~L}$ graphene in which silicene induces a band gap. Adapted from ref. 130 with permission from the American Chemical Society, Copyright 2020. (B) Illustration of the intercalation of $3 \mathrm{~L}$ graphene by $\mathrm{FeCl}_{3}$. Reproduced with from ref. 131 with permission from Wiley-VCH Verlag GmbH \& Co, Copyright 2020. 
subsequently intercalated by silicene (Fig. 18A). It is reported that the layer of silicene, which is intercalated between $\mathrm{Ru}$ and the $2 \mathrm{~L}$ of graphene induces a band gap of about $0.2 \mathrm{eV} .^{130} \mathrm{In}$ another approach 3L graphene, prepared by mechanical cleavage, was intercalated by $\mathrm{FeCl}_{3}$ by the two-zone method (Fig. 18B). Under the experimental conditions a stage 2 intercalation product is obtained, as evidenced by a splited $\mathrm{G}$ peak in the Raman spectrum. A band gap of $0.13 \mathrm{eV}$ was calculated for the experimentally obtained structure. In addition, the authors report that the intercalation compound is stable in ambient conditions. ${ }^{131}$ In general, the decomposition of $\mathrm{FeCl}_{3}$ intercalated graphite is kinetically hindered, since water must diffuse into the interlayers. It is described that $\left[\mathrm{FeCl}_{2}\left(\mathrm{OH}_{2}\right)_{4}\right]^{+}$and $4 \mathrm{Cl}^{-}$ are initially formed by the reaction of $4 \mathrm{H}_{2} \mathrm{O}$ and $\left[\mathrm{FeCl}_{6}\right]^{3-}$. Subsequently, $\left[\mathrm{FeCl}_{4}\right]^{-}$ions are formed, which are less densely packed. ${ }^{133}$

\subsection{Magnetism}

Among many important properties of 2DMs, the active magnetic response or magnetism has been studied for the development of various applications, including spill oil recovery, targeted drug delivery, and antibacterial interfaces. ${ }^{\text {134-137 }}$ Few-layered hosts with magnetic intercalants are unique systems to study magnetism in the 2D limit of materials. ${ }^{47,57,138} \mathrm{FeCl}_{3}$ has been intercalated into macroscopic scale $\left(1 \mathrm{~cm}^{2}\right)$ epitaxial $3 \mathrm{~L}$ graphene grown on $4 \mathrm{H}-\mathrm{SiC}$ to a stage 1 compound (Fig. 19A). ${ }^{57}$ The measured magnetoconductance shows a strong weak localization (WL) feature at cryogenic temperatures (<25 K, Fig. 19C). We note that similar WL in resistance have been reported in bulk magnetic-acceptor GICs $\left(\mathrm{FeCl}_{3}, \mathrm{CoCl}_{2}\right),{ }^{\mathbf{1 3 9}, \mathbf{1 4 0}}$ where the abrupt change of resistance has been connected with its magnetic transition. ${ }^{47}$

As shown in Fig. 19B, a detailed study of the temperature dependence of the longitudinal magnetoresistance shows that the WL peak is heavily suppressed when the temperature increases. These findings demonstrate that the intercalation of $\mathrm{FeCl}_{3}$ originates from single layer-like hole gases in the stacking with a phase coherence length $(L \phi)$ as large as $1.17 \pm 0.08 \mu \mathrm{m}$ at $280 \mathrm{mK}$. The temperature dependence of $L \phi$ shows a steep decrease for temperatures higher than $\sim 30 \mathrm{~K}$ (compatible with the $2 \mathrm{D}$ magnetic correlations in the plane of $\mathrm{FeCl}_{3}$ ), while $L \phi$ tends to saturate at temperatures lower than $\sim 4 \mathrm{~K}$ (compatible with 3D antiferromagnetic coupling between planes of $\left.\mathrm{FeCl}_{3}\right){ }^{57}$ For temperatures higher than $30 \mathrm{~K}$, a sharper decrease of $L \phi$ is observed in $\mathrm{FeCl}_{3}$-FLG, as compared to pristine graphene indicating that randomly oriented magnetic moments in the intercalated FLG are driving excessive dephasing (Fig. 19D). Ferromagnetism and superconductivity are two antagonistic phenomena since ferromagnetic exchange fields tend to destroy singlet Cooper pairs.

The coordination of these two competing phases has been achieved by alternative stacking of superconductor and ferromagnetic layers in vertical heterostructures. ${ }^{142,143}$ However, an interlayer-space-confined chemical design (ICCD) is reported to integrate these two phases in one single-atom-doped $\mathrm{TaS}_{2}$ layer, whereby ferromagnetism is observed in the superconducting

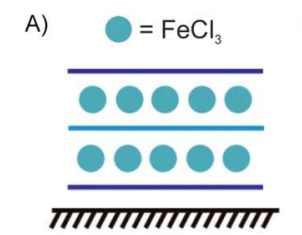

$4 \mathrm{H} \mathrm{SiC/G/FeCl} / \mathrm{G} / \mathrm{FeCl}_{3} / \mathrm{G}$
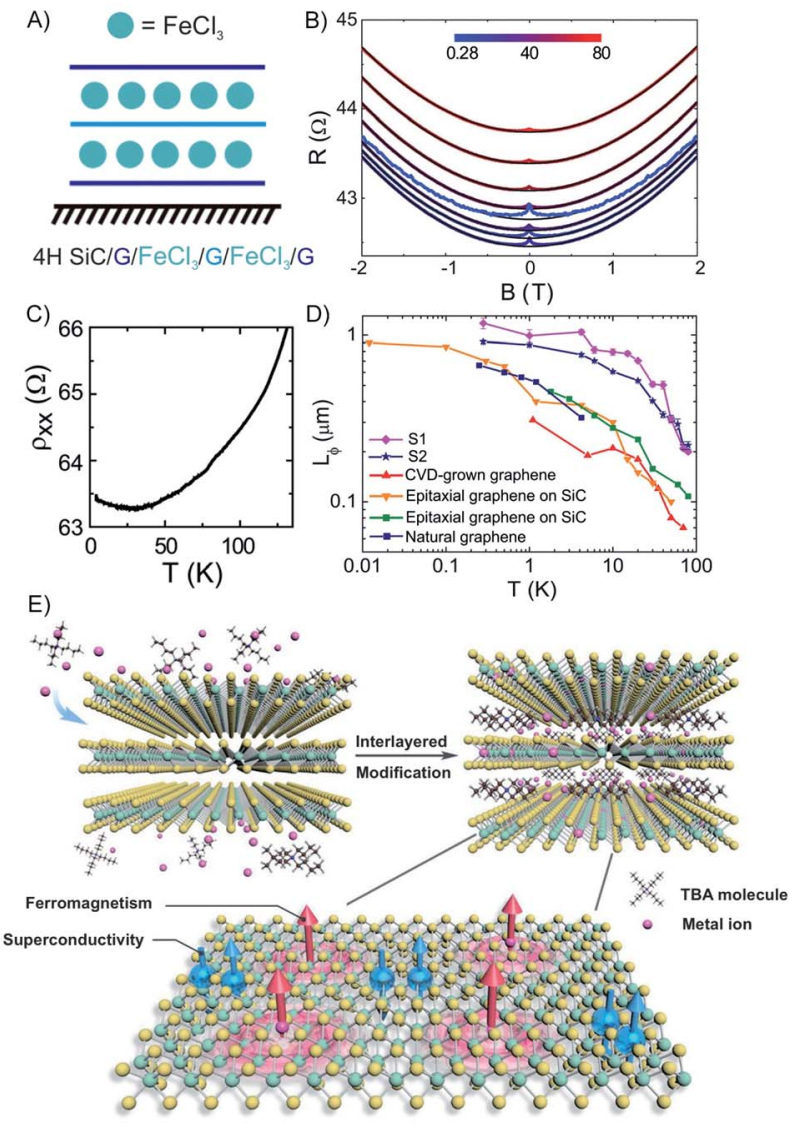

Fig. 19 (A) Scheme of $\mathrm{FeCl}_{3}$ intercalated 3L graphene. (B) The temperature dependence of the longitudinal resistivity. (C) Magneto resistance at various temperatures (see color-coded legend). (D) The temperature dependence of $L \phi$ for pristine graphene prepared by different methods. The values are compared to other works (original works are referenced in the cited publication) to the estimated values of $\mathrm{FeCl}_{3}-3 \mathrm{~L}$ graphene. Adapted from ref. 57 with permission from the American Chemical Society, Copyright 2014. (E) Illustration of the interlayer-space-confined chemical design toward the synthesis of $\mathrm{TaS}_{2}$ inorganic/organic molecular superlattice with the superconducting regions and ferromagnetic regions in single atomic layers. Reproduced from ref. 141 with permission from Wiley-VCH Verlag GmbH \& Co, Copyright 2020.

$\mathrm{TaS}_{2}$ layers. ${ }^{\mathbf{1 4 1}}$ The intercalation of bulky $2 \mathrm{H}-\mathrm{TaS}_{2}$ crystal with tetrabutylammonium chloride molecules expands its gap between layers, now suitable for single-atom doping via cointercalated Co ions, resulting in the formation of quasi-1L Co-doped $\mathrm{TaS}_{2}$ superlattices (Fig. 19E). Furthermore, Codoped $\mathrm{TaS}_{2}$ is exfoliated into ultrathin flakes $(20 \mathrm{~nm})$ under ultrasonication and dispersed in DMF, by which their magnetic properties were investigated. Isolated Co atoms are decorated in the basal plane of the $\mathrm{TaS}_{2}$ via replacing the Ta atom or anchoring at a hollow site, wherein the orbital-selected $\mathrm{p}^{-}$ $\mathrm{d}$ hybridization between Co and adjacent Ta and $\mathrm{S}$ atoms induces local magnetic moments with strong ferromagnetic coupling. This ICCD approach can be applied to intercalate various metal ions, enabling the synthesis of a series of crystalsize $\mathrm{TaS}_{2}$ molecular superlattices. 


\subsection{Optical properties}

4.4.1 Transparency. The combination of transparency and electrical conductivity of materials is the basis for touchscreen applications or organic light emitting devices. Most touch panels use indium tin oxide and the surface resistance is between 5-100 $\Omega \mathrm{sq}^{-1}$. Thereby, the transmittance at $550 \mathrm{~nm}$ is about $85 \%$. Reaching such values using $2 \mathrm{DMs}$ as single or multilayer is difficult. Graphene, as thinnest material absorbs $2.3 \%$ of light per layer and after chemical doping by e.g. TFSA $\left(\left(\mathrm{CF}_{3} \mathrm{SO}_{2}\right)_{2} \mathrm{NH}\right)$ the resistance dropped to $129 \Omega \mathrm{sq}^{-1} \cdot{ }^{\mathbf{1 4 4}}$ With stacking four layers of graphene and doping by $\mathrm{AuCl}_{3}$ the sheet resistance can be reduced to $34 \Omega \mathrm{sq}^{-1} \cdot{ }^{145}$ However, intercalation of few-layer graphene, such as $4 \mathrm{~L}$ or $5 \mathrm{~L}$ was demonstrated to reduce the sheet resistance keeping the transparency high. ${ }^{58}$ Thus, exfoliated few-layers, such as 5L graphene, were intercalated by $\mathrm{FeCl}_{3}$ at $360{ }^{\circ} \mathrm{C}$ using the two-zone method. The realized sheet resistance for intercalated $5 \mathrm{~L}$ graphene was determined to $8.8 \Omega \mathrm{sq}^{-1}$ at an optical transmittance of about $84 \% .^{58}$ Large area graphene films $\left(11 \times 11 \mathrm{~cm}^{2}\right)$ were fabricated by artificial layer-by-layer transfer of $\mathrm{AuCl}_{3}$ doped graphene. The sheet resistance of layer-by-layer $\mathrm{AuCl}_{3}$ doped $4 \mathrm{~L}$ graphene was $54 \Omega \mathrm{sq}^{-1}$ at $85 \%$ transmittance. This method offers not only an improvement of sheet resistance and uniformity but also better environmental stability compared to topmost layer doping. ${ }^{\mathbf{1 4 6}}$

Metallization of graphite and few-layer graphene, respectively, is possible by intercalation with $\mathrm{Li}, \mathrm{K}$ or $\mathrm{Cs}$ as intercalant. ${ }^{38,49}$ With intercalation the Fermi level is shifted to higher energy. Accordingly, interband optical transitions are suppressed, which can be explained by Pauli blocking. Thus, the transmittance is increased and reaches the visible at high
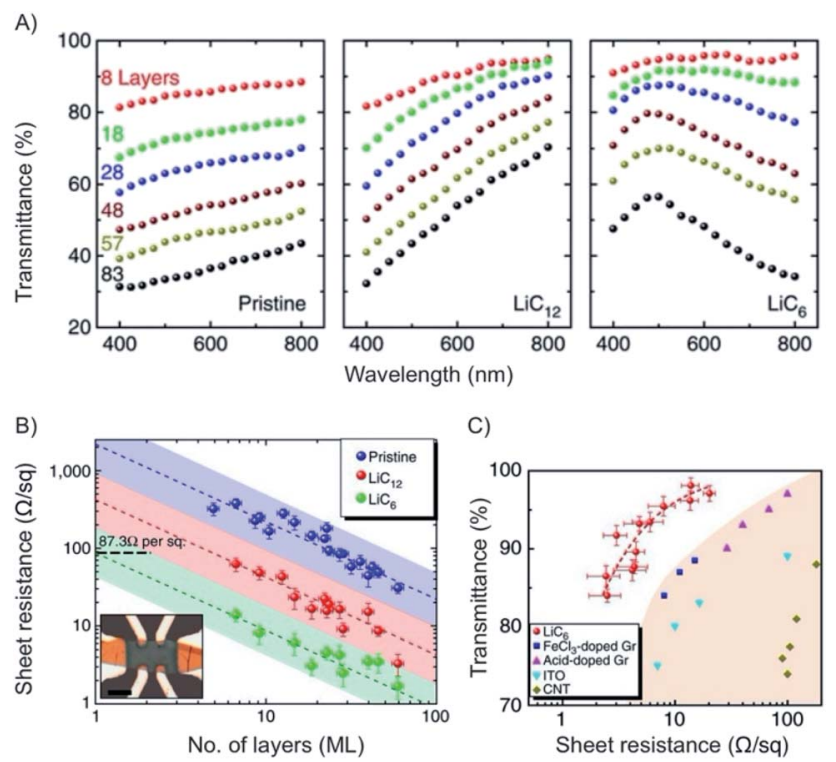

Fig. 20 (A) Plots of transmittance vs. wavelength for few- and multilayers of graphene and intercalated materials. (B) Plot of the sheet resistance vs. number of layers of intercalated materials. (C) Plot of transmittance vs. sheet resistance for intercalated materials and other reference materials. Reprinted from ref. 148 with permission from Macmillan Publishers Ltd: Nature Communications, Copyright 2014. charge-carrier densities. ${ }^{\mathbf{1 4 7}}$ Since those optical transitions are no longer possible, as those states are filled by the heavy n-doping.

With n-doping of graphene the charge carrier density increases to about $6 \times 10^{14} \mathrm{~cm}^{-2}$ and therefore the conductivity. The Fermi energy increases to about $1.5 \mathrm{eV} \cdot{ }^{\mathbf{1 4 9}}$ This concept was studied in situ for few- and multilayer graphene. ${ }^{\mathbf{1 4 8}}$ As depicted in Fig. 20, a maximum in transmittance is reached at $500 \mathrm{~nm}$ for multi-layers of graphene, such as $19 \mathrm{~L}$ with transmission of $91.7 \%$. The results of $e . g$. few-layer graphene (8L) are also shown in Fig. 20.

4.4.2 Optoelectronic properties. Few-layered 2DMs and their heterostructures have shown great promise for new optoelectronic applications, ${ }^{\mathbf{1 5 0 , 1 5 1}}$ such as high-speed optical communications ${ }^{152,153}$ and wide-optical-bandwidth photodetectors. ${ }^{154,155}$ Graphene has attracted intense research due to its atomic layer thickness, ultrahigh strength and free carrier mobility. Nevertheless, the weak light absorption and zero bandgap of graphene largely limited its application in the optoelectronic field. Thus, graphene-based intercalated structures have been proposed to improve the low optical absorption and quantum efficiency of graphene. Fano-resonant Au plasmonic clusters have been sandwiched between $2 \mathrm{~L}$ graphene producing a photodetector, where two graphene layers perform as two carrier channels. The photocurrent of the device was enhanced 8 times, which is owing to the hot electrons transfer generated in antenna structure and direct plasmon-enhanced excitation of intrinsic graphene electrons. The internal quantum efficiency for the device achieved up to $20 \%$ in the visible and near-infrared regions of the spectrum. ${ }^{156}$ As a lowcost and easy-accessible alternative, rhodamine 6G (R6G) dye with only $1 \mathrm{~L}$ thickness $(0.85 \mathrm{~nm})$ was deposited by a simple dip-

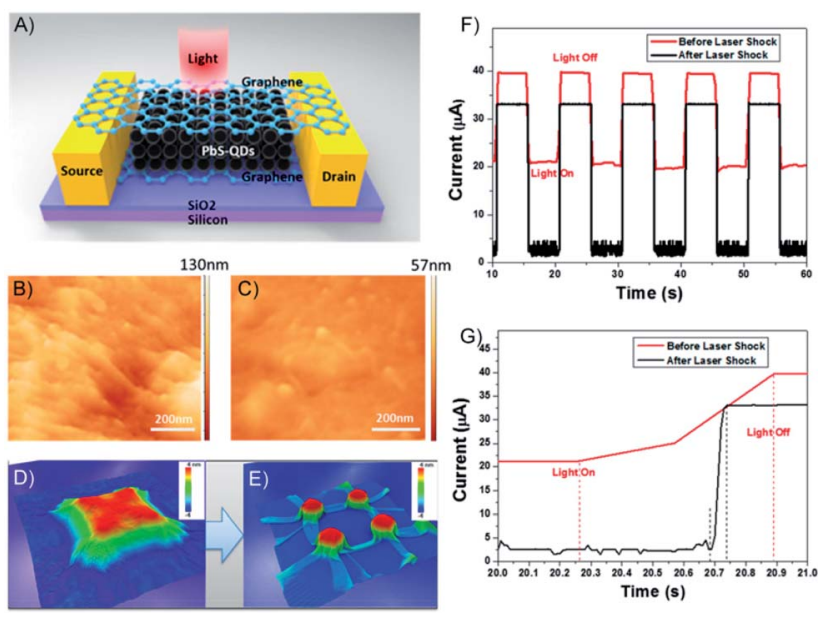

Fig. 21 (A) Structure of graphene/PbS/graphene hybrid structures. The channel length between source and drain is $50 \mu \mathrm{m}$. AFM image of graphene wrapped on PbS QDs (B) before and (C) after laser shock fabrication. Molecular dynamics simulation results demonstrate graphene wrapping on 3D feature surface (D) before and (E) after the laser shock process. (F) I vs. $t$ curve of graphene/PbS/graphene hybrid photosensor before and after the laser shock process at the gate voltage of $20 \mathrm{~V}$ and (G) magnified image of (F). Adapted from ref. 158 with permission from the American Chemical Society, Copyright 2017. 


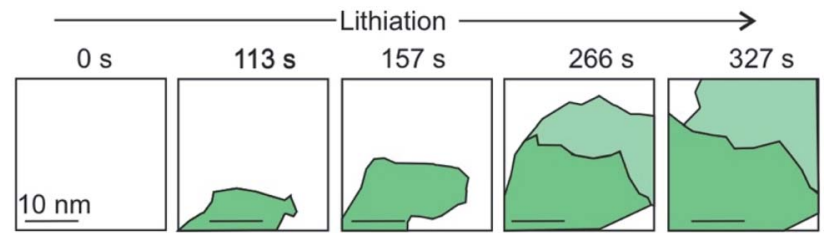

Fig. 22 A schematic and simplified illustration of the temporal evolution of lithium intercalation into $2 \mathrm{~L}$ graphene as evaluated by transmission electron microscopy. Crystal grains are color-coded by their in-plane orientation in green and light green. More details can be found in the original figure. ${ }^{15}$

coating method to build a graphene-dye-graphene (G-R-G) sandwich photodetector. ${ }^{157}$ The strong $\pi-\pi$ interaction force in the $\mathrm{G}-\mathrm{R}-\mathrm{G}$ structure reduced the intermolecular distance, which accelerated the photoexcited charge transfer from the top and bottom graphene to the R6G $1 \mathrm{~L}$. The photocurrent and responsivity of the G-R-G device was found to be $\sim 40$ times better than R6G-attached single-graphene device. ${ }^{157}$ However, traditional transfer methods restrict the contact between the top layer of graphene and the underlying intercalant (especially for $0 \mathrm{D}$ and $3 \mathrm{D}$ intercalant) to grid-space contact, resulting in a weaker transmission in the structure and inevitable artificial scattering. To solve the problem, a graphene/PbS-quantum dots (PbS-QDs)/graphene sandwich structure with seamless 2D/0D contact was fabricated by laser shock imprinting, which optomechanically tunes the morphology of 2DMs to perfectly wrap on $0 \mathrm{D}$ materials and efficiently collect carriers from the PbSQDs (Fig. 21A-E). This seamless integrated 2D/0D/2D structure significantly enhanced the carrier transmission, double increase of photoresponse, 20 -fold response time and 13-fold photoresponse speed (Fig. 21F and G). ${ }^{158}$

The valley degree of freedom in 2D-crystals recently emerged as a novel information carrier in addition to spin and charge applications. ${ }^{159}$ TMDC-1Ls feature a coupled spin-valley degree of freedom and have emerged as an exciting spin/valleytronic platform. However, the intralayer exciton spin/valley lifetime in $1 \mathrm{~L}$ TMDCs is limited to tens of nanoseconds due to the unique spin-valley locking behaviour. Achieving long-lived population and polarization lifetimes in TMDC materials is of central importance for their optoelectronic, photonic, and spin/ valleytronic applications.

Type II heterostructures, such as $\mathrm{WSe}_{2} / \mathrm{MoSe}_{2}$ have been fabricated to reach long valley polarization times, but precise control of the exciton transformation process (including intralayer to interlayer exciton transition and recombination) and a valley polarization process via structural tuning is more challenging. An intermediate layer of hBN was transferred between a $\mathrm{WSe}_{2} / \mathrm{WS}_{2}$ heterostructure. The increased spatial separation with hBN intercalation weakens the electron-hole Coulomb interaction and significantly prolongs the interlayer exciton population and valley relaxation lifetime in vdW heterostructures. ${ }^{160}$ Therefore, $\mathrm{WSe}_{2} / \mathrm{WS}_{2}$ heterostructures with $1 \mathrm{~L}$ hBN intercalation exhibit a hole valley polarization lifetime of $\sim 60$ ps at room temperature, which is approximately threefold and 3 orders of magnitude longer than that in $\mathrm{WSe}_{2} / \mathrm{WS}_{2}$ hetero$2 \mathrm{~L}$ without $\mathrm{hBN}$ and $\mathrm{WSe}_{2}-1 \mathrm{~L}$, respectively. ${ }^{160}$

\subsection{Energy storage}

The intercalation of Li ions into the galleries of a graphite anode is the key step of electrical energy storage in Li-ion batteries that are the major energy storage technology from electric cars to mobile devices. The ideal energy storage device must combine a high energy and power density with a long cycling lifetime. The energy density depends on the amount of charge that can be stored in the material. The power density depends on how fast the intercalant can diffuse into and out of the layered material. These two factors should be combined with a high cycling life time, that is a large number of intercalation/ deintercalation cycles without significant performance loss. For the further development of this key technology a thorough understanding of the processes at the atomic level is highly desired. Studying the intercalation at the 2D level allows to follow the electrochemical intercalation process by various techniques in situ such as XRD, ${ }^{161}$ Hall measurements ${ }^{22,93}$ and also to follow the process by optical microscopy ${ }^{89,162}$ and even transmission electron microscopy at atomic resolution. ${ }^{15,93,163}$ TEM imaging not only allows to optically follow the intercalation and deintercalation of the ions into the few-layered 2DM, but also identifies areas of varying crystallinity and grain sizes (Fig. 22). As highlighted in chapter 1, Kühne et al. have demonstrated the $\mathrm{Li}$ intercalation of $2 \mathrm{~L}$ and $3 \mathrm{~L}$ graphene deviating from the expected $\mathrm{C}_{6} \mathrm{LiC}_{6}$ compound structure using TEM imaging at atomic resolution. The increased Li storage capacity by super dense ordering of $\mathrm{Li}$ in the vdW gap of $2 \mathrm{~L}$ graphene compared to its bulk counterpart is attributed to the fact that

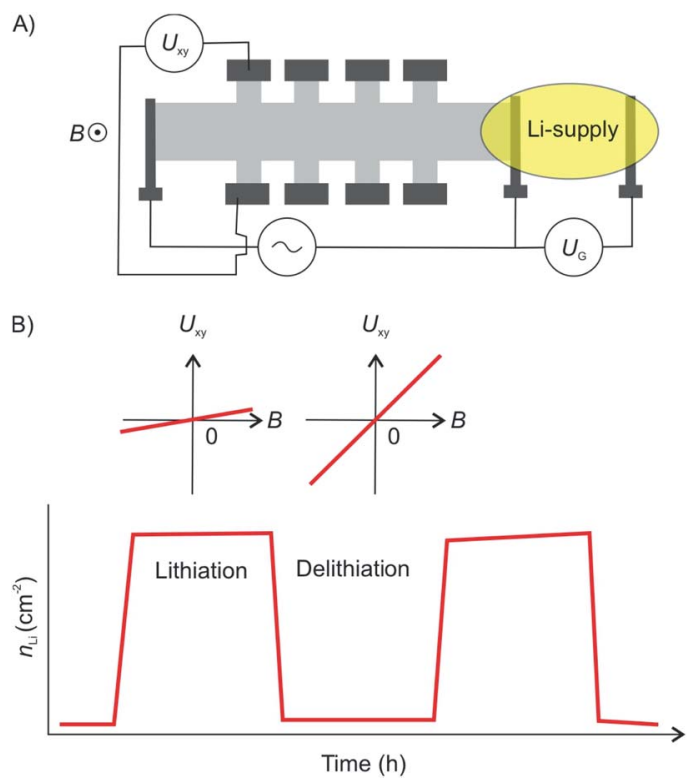

Fig. 23 (A) Illustration the $2 \mathrm{~L}$ graphene device for measurement of Liion diffusion rates. The $2 \mathrm{~L}$ device was shaped into a Hall bar configuration. (B) Lithiation/delithiation cycles visualized by charge carrier concentration extracted from Hall measurements showing full reversibility of the intercalation cycles. ${ }^{93}$ 
the $2 \mathrm{~L}$ spread more easily when they are isolated from their bulk crystal. This finding indicates distinct Li storage arrangements with larger storage capacities in $2 \mathrm{~L}$ compounds compared to their bulk counterparts.

The diffusion of the intercalant into the layered material is an important parameter as it defines the speed at which a device can be charged and discharged. The diffusion rate of $\mathrm{Li}$ ions into graphite is relatively low at $10^{-8} \mathrm{~cm}^{2} \mathrm{~s}^{-1}$ resulting in a low power density of Li-ion batteries. By thinning down the electrode to $2 \mathrm{~L}$ of graphene an exceptional diffusion rate of up to 7 $\times 10^{-5} \mathrm{~cm}^{2} \mathrm{~s}^{-1}$ was reported. ${ }^{93}$ The diffusion rate was determined by measuring the temporal evolution of the Li density at discrete positions in the device using Hall measurements (Fig. 23A and B). The increased diffusion rate is attributed to the widening of the vdW gap by the intercalated Li ions. Again, this effect can be attributed to the fact that the isolated layers may spread more easily compared to their bulk counterparts.

As a general scheme, tuning the interlayer distance in $2 \mathrm{~L}$ materials can be used to engineer the energy storage properties. This can be achieved by stacking different 2DMs, creating vdW heterostructures. The intercalation of heterostructures of 2DMs with dissimilar properties may be a promising way to develop high-performance energy storage devices with increasing complexity. The combination of 2DMs allows to make use of the materials advantages by eliminating some of their shortcomings by creating new heterointerfaces and combining the materials electronic properties. For example, the expansion of an electrode upon intercalation is a crucial factor for the cycling lifetime of a battery device. A heterostructure of multi-layered $\mathrm{VOPO}_{4}$ with multilayer graphene showed negligible expansion upon electrochemical intercalation and deintercalation with $\mathrm{Na}^{+}, \mathrm{K}^{+}, \mathrm{Zn}^{2+}, \mathrm{Al}^{3+}$ cations that was attributed to the in-plane lattice mismatch and the resulting compressive strain on the $\mathrm{VOPO}_{4}$ sheets. ${ }^{164}$ As the number of $2 \mathrm{DMs}$ is still increasing they are covering a wide range of electronic properties from metallic materials such as $\mathrm{Ti}_{3} \mathrm{C}_{2}$, zero-band gap as in the case of graphene, semiconducting such as $\mathrm{MoS}_{2}$ or black phosphorous to large band gap materials such as hBN. The combination of these materials creates a large library of new materials that allow to tune their electrode properties in energy storage devices. $^{165}$

Mechanical strain induced by the lattice mismatch in vdW heterostructures has been demonstrated in multi-layered materials to control various materials properties. ${ }^{\mathbf{1 6 6}}$ Mechanical strain induced by the lattice mismatch of vertically stacked multi-layered $\mathrm{MoS}_{2}$ and thin carbon layers was utilized to influence the pathways of electrochemical reactions upon $\mathrm{Li}$ intercalation. By engineering the strain in the system the chemical reaction could be influenced from intercalation in the pristine $\mathrm{MoS}_{2}$ system to the chemical conversion to Lithium sulfide in the case of thin carbon layers stacked $\mathrm{MoS}_{2}$ multilayers. ${ }^{86}$ On the $2 \mathrm{~L}$ level, the $\mathrm{Li}$ intercalation in vdW heterostructures of graphene and molybdenum dichalcogenide $\left(\mathrm{MoX}_{2}, \mathrm{X}=\mathrm{S}\right.$, Se) encapsulated in hBN showed a 10 -fold increase of accumulated charge compared to $\mathrm{MoS}_{2} / \mathrm{MoS}_{2} 2 \mathrm{~L}$ devices as demonstrated by Bediako et al. ${ }^{87}$ They fabricated five vdW heterostructures of graphene, TMDCs and hBN in which

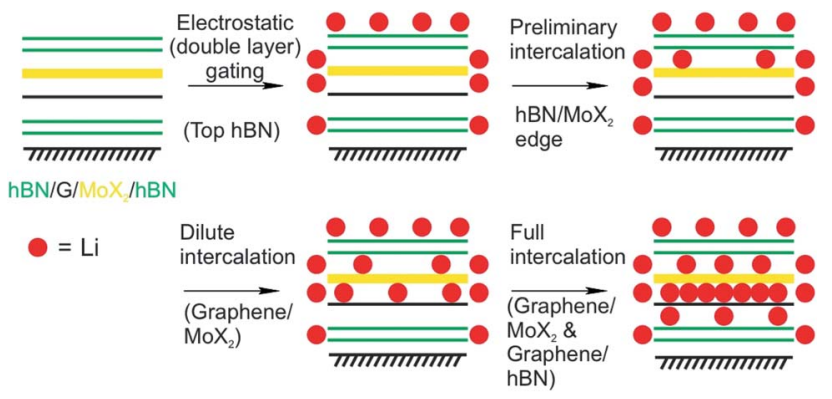

Fig. 24 Proposed four-step Li intercalation mechanism for a hBN (green)/MoX 2 (yellow)/graphene (black)/hBN heterostructure, evidenced by in situ HRTEM imaging. ${ }^{87}$

graphene and $\mathrm{MoX}_{2}$ layers are encapsulated by $\mathrm{hBN}$ and employed as the working electrode of an electrochemical cell. By using the Hall potentiometry method they followed the $\mathrm{Li}$ intercalation upon sweeping the potential in various heterostructures. They elucidated the mechanisms of the Li intercalation at the various heterointerfaces. By combining the results of transmission electron microscopy, in situ magnetoresistance, optical spectroscopy techniques, ab initio calculations and lowtemperature quantum magneto-oscillation measurements they derived an intercalation mechanism that describes the intercalation into a graphene/ $\mathrm{MoX}_{2} 2 \mathrm{~L}$ encapsulated by hBN (Fig. 24). The proposed mechanism showcases the varying intercalation processes at the heterointerfaces of the 2DMs that can be understood by the Li binding energies calculated for the various heterointerfaces. Their study revealed that the capacity of the graphene/ $\mathrm{MoX}_{2}$ heterointerface is more than 10-times larger than the capacity of the other heterointerfaces (graphene/ hBN, $\left.\mathrm{MoX}_{2} / \mathrm{hBN}\right)$. Furthermore, they observed a decreased onset intercalation voltage of graphene/ $\mathrm{MoX}_{2}$ that is about $0.5 \mathrm{~V}$ larger than that of a graphene/hBN heterostructure shown in a previous study. ${ }^{22} \mathrm{MoS}_{2}$ undergoes a phase transition from the initial semiconducting $\mathrm{H}$-phase to the metallic $\mathrm{T}^{\prime}-\mathrm{MoS}_{2}$ phase after charge transfer from Li. This phase transition lowers the activation barrier for further $\mathrm{Li}$ intercalation.

\subsection{Chemical reactions}

The process of chemical reactions takes basically place on the surface of a bulk material. By thinning down the material, properties change such as the reactivity or absorption and emission properties for example in QDs, as outlined below. Not only by increasing the surface, but also by intercalation, layers become more accessible to the reactant or are activated by the intercalant. In principle, chemical reactions can be accelerated or observed for the first time in intercalated structures.

One of the first heterostructures with mixed dimensional materials $(2 \mathrm{D} / 0 \mathrm{D} / 2 \mathrm{D})$ consists of a graphene-coated TEM grid with thermally evaporated fullerenes and transferred graphene as top-layer (Fig. 25A and B). ${ }^{101}$ Due to the low fullerene moving barrier of $5 \mathrm{meV}$, the self-assembly of the 0D component leads to big areas of single-layered, hexagonally closed packaging in between graphene. It turned out that the lattice spacing is $4-5 \%$ 


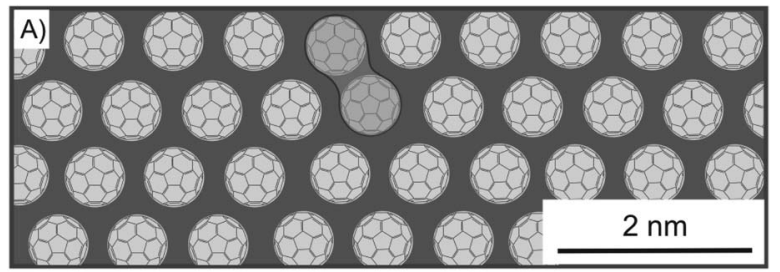

B)

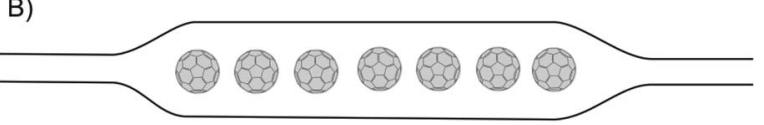

C)

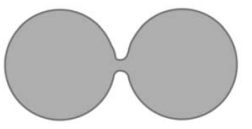

D)

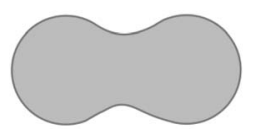

Fig. 25 Fullerenes encapsulated between graphene layers and investigated by STEM analysis. (A) Illustration of a $1 \mathrm{~L}$ of hexagonally ordered fullerenes, showing a bonded couple. (B) Schematic illustration of the heterostructure of graphene/ $C_{60} / g r a p h e n e$. (C) Illustration of a loosely bound fullerene dimer and (D) of a fullerene peanut dimer. ${ }^{101}$

smaller than in equivalent 3D crystallites, while the fullerenes remain rotationally active. Although graphene acts as a protecting layer during STEM, the e-beam irradiation activates the molecules and causes bond formation between fullerenes forming a peanut-like dimer, as shown in Fig. 25A and D, a process which is supported by calculations starting from a loosely bound dimer of fullerenes (Fig. 25C). Due to the regular structure of the graphene lattices, those lattices can be filtered out in TEM images, enabling the unhindered observation and study of the intercalant. The encapsulation within graphene can also be applied to other 2DM, as demonstrated for $\mathrm{MoS}_{2}{ }^{167}$ With this approach, molecular structures and their dynamics can be analyzed.

The factor for improving reactivity using the concept of heterostructures is significant for QDs. ${ }^{168}$ QDs gained attention in photocatalysis for their high quantum yields and specific quantum confinement, but lacked in photostability, long lifetime and fast electron transfer. However, Huang et al. fabricated a heterostructure that did overcome those drawbacks (Fig. 26C). ${ }^{168}$ The heterostructure is fabricated layer-by-layer, by electrostatic self-assembly in aqueous solution, with positively charged graphene oxide, which was functionalized by ethylenediamine (Fig. 26A), and negatively charged CdSe QDs, modified by sulfanylacetic acid (Fig. 26B) connecting graphene oxide layers and QDs by peptide bonds. Subsequently, reduction generates reduced graphene oxide, which is further termed as graphene. It was shown that graphene improves the photostability, adsorption of reactants and the separation of excitons of QDs due to fast electron transport.

The oxidation of aromatic alcohols to aldehydes (Fig. 26D), such as benzyl alcohol to benzaldehyde, and the reduction of nitroaromatics to amino aromatics, here to aniline (Fig. 26E), are enhanced compared to mere QDs, because the reactivity is ascribed to holes and electrons as well as hydroxyl radicals. The
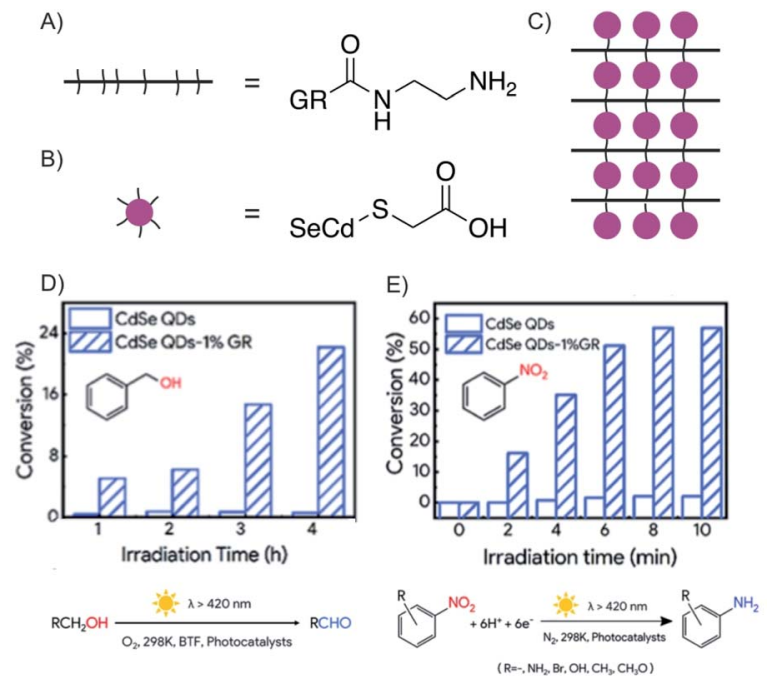

Fig. 26 Enhanced electron transfer in covalent bonded CdSe QDs/ reduced graphene oxide (GR) heterostructure obtaining enhanced photoredox conversion. (A) Schematic and chemical structure of ethylenediamine functionalized graphene. (B) Schematic and chemical structure of sulfanylacetic acid functionalized CdSe QDs. (C) Schematic illustration of CdSe QDs/reduced graphene oxide heterostructure. (D) Comparison of conversion efficiency of the heterostructure and the sole QD for the oxidation of benzyl alcohol to the aldehyde. (E) Comparison of the conversion efficiency of the heterostructure and the sole QD for the reduction of nitrobenzene to aniline. Adapted from ref. 168 with permission from the American Chemical Society, Copyright 2019.

heterostructure in this example possesses enhanced photoredox activity due to the artificial intercalation of QD between graphene.

\section{Perspectives}

We presented the emerging field of research of the intercalation of $2 \mathrm{DMs}$ with a special focus on intercalated $2 \mathrm{~L}$ and $3 \mathrm{~L}$ systems. As described in the last Section 4.6, a heterostructure assembled of a $2 \mathrm{DM}$ and a photosensitizer is promising for developing photocatalysts. However, the self-assembly strategy used in that example does not stop at the thinnest intercalated counterpart to the bulk but leads to 3D structures. Although not investigated yet, intercalated $2 \mathrm{~L}$ systems bear a larger area for the interaction with substrate molecules and will possibly lead to higher quantum yields and thus, more efficiency. With thinning intercalated 2DMs the ultimate surface access is possible, in particular once intercalated few-layered 2DMs are placed on a membrane support, giving access to both sides. Furthermore, manipulating the intercalant can be highly effective in such systems, since only one layer of a $2 \mathrm{DM}$ is shielding the intercalant. Therefore, we speculate that novel concepts for sensing devices will emerge and unforeseen properties will be found, as already described for $1 \mathrm{~L}$ or $2 \mathrm{~L}$ graphene and the $2 \mathrm{DMs}$ family in general. Another benefit of thinning materials to the limit is the ability to observe elemental processes by spectroscopy probing the complete system compared to the surface as only a minor 
part of the bulk, e.g. by Raman spectroscopy. Moreover, microscopes improve and can monitor intercalation processes on the atomic scale, leading to a better understanding of even application relevant charge storage systems. Assembly techniques are already established to fabricate complex layered systems, which are based on 2DMs as well as molecules, respectively. At the same time, different scientific fields come together and envision novel applications.

In this review, we focused on the fields of superconductivity, band gap tuning, magnetic ordering, optical properties, energy storage and chemical reactions. However, with advancing the field of intercalated few-layered 2DMs more functional systems with a defined focus on specific applications will emerge. Possible highlights in sensing applications can be imagined since it is already a highly active field of research relevant for all kinds of science, e.g. for sensing of small environmental molecules, monitoring biologically relevant species in cells, light sensors or mechanical force sensors, to mention only some. Finally, with increasing the understanding of intercalated 2DMs, it will be possible to gain more control over the chemistry, which can be induced close to the surface. Thus, there is a good chance to develop more energy-efficient chemical reactions with even accelerated kinetics and specificity.

\section{Conflicts of interest}

There are no conflicts to declare.

\section{Acknowledgements}

We appreciate financial support by the German Research Foundation (DFG, grant no. 392444269). This research is supported by the China Scholarship Council (CSC).

\section{Notes and references}

1 Angew. Chem., Int. Ed., 2019, 58, 16723, https:// onlinelibrary.wiley.com/doi/epdf/10.1002/anie.201912886.

2 A. K. Geim, Angew. Chem., Int. Ed., 2011, 50, 6966-6985.

3 S. Eigler and A. Hirsch, Angew. Chem., Int. Ed., 2014, 53, 7720-7738.

4 A. Hirsch and F. Hauke, Angew. Chem., Int. Ed., 2018, 57, 4338-4354.

5 J. Sturala, Z. Sofer and M. Pumera, Angew. Chem., Int. Ed., 2019, 58, 7551-7557.

6 K. S. Subrahmanyam, A. K. Manna, S. K. Pati and C. N. R. Rao, Chem. Phys. Lett., 2010, 497, 70-75.

7 B. Cai, S. Zhang, Z. Yan and H. Zeng, ChemNanoMat, 2015, 1, 542-557.

8 P. Miró, M. Audiffred and T. Heine, Chem. Soc. Rev., 2014, 43, 6537-6554.

9 K. S. Novoselov, A. Mishchenko, A. Carvalho and A. H. Castro Neto, Science, 2016, 353, aac9439.

10 W. Rüdorff and U. Hofmann, Z. Anorg. Allg. Chem., 1938, 238, 1-50.

11 C. Schafhaeutl, London, Edinburgh Dublin Philos. Mag. J. Sci., 2009, 16, 570-590.
12 M. S. Dresselhaus and G. Dresselhaus, Adv. Phys., 1980, 30, 139-326.

13 A. T. S. Wee, ACS Nano, 2012, 6, 5739-5741.

14 J. Wang, F. Ma and M. Sun, RSC Adv., 2017, 7, 16801-16822.

15 M. Kühne, F. Börrnert, S. Fecher, M. Ghorbani-Asl, J. Biskupek, D. Samuelis, A. V. Krasheninnikov, U. Kaiser and J. H. Smet, Nature, 2018, 564, 234-239.

16 M. S. Stark, K. L. Kuntz, S. J. Martens and S. C. Warren, Adv. Mater., 2019, 31, 1808213.

17 J. Wan, S. D. Lacey, J. Dai, W. Bao, M. S. Fuhrer and L. Hu, Chem. Soc. Rev., 2016, 45, 6742-6765.

18 L. Daukiya, M. N. Nair, M. Cranney, F. Vonau, S. HajjarGarreau, D. Aubel and L. Simon, Prog. Surf. Sci., 2019, 94, 1-20.

19 Y. Li, Y. Lu, P. Adelhelm, M.-M. Titirici and Y.-S. Hu, Chem. Soc. Rev., 2019, 48, 4655-4687.

20 A. Lerf, Dalton Trans., 2014, 43, 10276-10291.

21 A. Jain, P. Bharadwaj, S. Heeg, M. Parzefall, T. Taniguchi, K. Watanabe and L. Novotny, Nanotechnology, 2018, 29, 265203.

22 S. Y. F. Zhao, G. A. Elbaz, D. K. Bediako, C. Yu, D. K. Efetov, Y. Guo, J. Ravichandran, K.-A. Min, S. Hong, T. Taniguchi, K. Watanabe, L. E. Brus, X. Roy and P. Kim, Nano Lett., 2018, 18, 460-466.

23 S. Eigler, Chem.-Eur. J., 2016, 22, 7012-7027.

24 D. T. Larson, I. Fampiou, G. Kim and E. Kaxiras, J. Phys. Chem. C, 2018, 122, 24535-24541.

25 L. B. Ebert, Annu. Rev. Mater. Sci., 1976, 6, 181-211.

26 D. E. Nixon and G. S. Parry, J. Phys. D: Appl. Phys., 1968, 1, 291-298.

27 T. Sasa, Y. Takahashi and T. Mukaibo, Carbon, 1971, 9, 407416.

28 D. E. Wessbecher, W. C. Forsman and J. R. Gaier, Synth. Met., 1988, 26, 185-194.

29 J. G. Hooley, W. P. Garby and J. Valentin, Carbon, 1965, 3, 716.

30 J. G. Hooley, Materials Science and Engineering, 1977, 31, 1724.

31 M. J. Rosseinsky, J. Mater. Chem., 1995, 5, 1497-1513.

32 Y. Iwai, M. Hirose, R. Kano, S. Kawasaki, Y. Hattori and K. Takahashi, J. Phys. Chem. Solids, 2008, 69, 1199-1202.

33 A. Sumiyoshi, H. Hyodo and K. Kimura, J. Phys. Chem. Solids, 2010, 71, 569-571.

34 R. B. Somoano, V. Hadek and A. Rembaum, J. Chem. Phys., 1973, 58, 697-701.

35 F. J. Di Salvo, G. W. Hull Jr, L. H. Schwartz, J. M. Voorhoeve and J. V. Waszczak, J. Chem. Phys., 1973, 59, 1922-1929.

36 D. Dumchenko, C. Gherman, L. Kulyuk, E. Fortin and E. Bucher, Thin Solid Films, 2006, 495, 82-85.

37 M. G. Alexander, D. P. Goshorn and D. G. Onn, Phys. Rev. B: Condens. Matter Mater. Phys., 1980, 22, 4535-4542.

38 M. H. Whangbo, W. Liang, J. Ren, S. N. Magonov and A. Wawkuschewski, J. Phys. Chem. C, 1994, 98, 7602-7607.

39 S. A. Solin and H. Zabel, Adv. Phys., 1988, 37, 87-254.

40 N. Emery, C. Hérold, J.-F. Marêché and P. Lagrange, Sci. Technol. Adv. Mater., 2008, 9, 044102. 
41 W. Krone, G. Wortmann and G. Kaindl, Synth. Met., 1989, 29, 247-252.

42 J. G. Hooley and M. Bartlett, Carbon, 1967, 5, 417-422.

43 J. G. Hooley, Carbon, 1973, 11, 225-236.

44 L. Salamanca-Riba, G. Roth, J. M. Gibson, A. R. Kortan, G. Dresselhaus and R. J. Birgeneau, Phys. Rev. B: Condens. Matter Mater. Phys., 1986, 33, 2738-2748.

45 C. Zeller, L. A. Pendrys and F. L. Vogel, J. Mater. Sci., 1979, 14, 2241-2248.

46 A. R. Beal and W. Y. Liang, Philos. Mag., 2006, 27, 13971416.

47 N. Kim, K. S. Kim, N. Jung, L. Brus and P. Kim, Nano Lett., 2011, 11, 860-865.

48 M. E. Huster, P. A. Heiney, V. B. Cajipe and J. E. Fischer, Phys. Rev. B: Condens. Matter Mater. Phys., 1987, 35, 33113326.

49 N. Jung, B. Kim, A. C. Crowther, N. Kim, C. Nuckolls and L. Brus, ACS Nano, 2011, 5, 5708-5716.

50 N. Jung, N. Kim, S. Jockusch, N. J. Turro, P. Kim and L. Brus, Nano Lett., 2009, 9, 4133-4137.

51 N. Jung, A. C. Crowther, N. Kim, P. Kim and L. Brus, ACS Nano, 2010, 4, 7005-7013.

52 J. G. Hooley, Carbon, 1972, 10, 155-163.

53 C.-J. Shih, A. Vijayaraghavan, R. Krishnan, R. Sharma, J.-H. Han, M.-H. Ham, Z. Jin, S. Lin, G. L. C. Paulus, N. F. Reuel, Q. H. Wang, D. Blankschtein and M. S. Strano, Nat. Nanotechnol., 2011, 6, 439-445.

54 D. J. Wehenkel, T. H. Bointon, T. Booth, P. Bøggild, M. F. Craciun and S. Russo, Sci. Rep., 2015, 5, 7609.

55 D. Zhan, L. Sun, Z. H. Ni, L. Liu, X. F. Fan, Y. Wang, T. Yu, Y. M. Lam, W. Huang and Z. X. Shen, Adv. Funct. Mater., 2010, 20, 3504-3509.

56 W. Zhao, P. H. Tan, J. Liu and A. C. Ferrari, J. Am. Chem. Soc., 2011, 133, 5941-5946.

57 T. H. Bointon, I. Khrapach, R. Yakimova, A. V. Shytov, M. F. Craciun and S. Russo, Nano Lett., 2014, 14, 1751-1755.

58 I. Khrapach, F. Withers, T. H. Bointon, D. K. Polyushkin, W. L. Barnes, S. Russo and M. F. Craciun, Adv. Mater., 2012, 24, 2844-2849.

59 W. S. Hummers Jr and R. E. Offeman, J. Am. Chem. Soc., 1958, 80, 1339.

60 S. Eigler, Commun. Chem., 2015, 51, 3162-3165.

61 S. Seiler, C. E. Halbig, F. Grote, P. Rietsch, F. Börrnert, U. Kaiser, B. Meyer and S. Eigler, Nat. Commun., 2018, 9, 836.

62 W. J. Zhao, P. H. Tan, J. Zhang and J. Liu, Phys. Rev. B: Condens. Matter Mater. Phys., 2010, 82, 245423.

63 A. Das, B. Chakraborty, S. Piscanec, S. Pisana, A. K. Sood and A. C. Ferrari, Phys. Rev. B: Condens. Matter Mater. Phys., 2009, 79, 155417.

64 K. J. Koski, C. D. Wessells, B. W. Reed, J. J. Cha, D. Kong and Y. Cui, J. Am. Chem. Soc., 2012, 134, 13773-13779.

65 K. J. Koski, J. J. Cha, B. W. Reed, C. D. Wessells, D. Kong and Y. Cui, J. Am. Chem. Soc., 2012, 134, 7584-7587.

66 K. P. Chen, F. R. Chung, M. Wang and K. J. Koski, J. Am. Chem. Soc., 2015, 137, 5431-5437.
67 Y. Gong, H. Yuan, C.-L. Wu, P. Tang, S.-Z. Yang, A. Yang, G. Li, B. Liu, J. van de Groep, M. L. Brongersma, M. F. Chisholm, S.-C. Zhang, W. Zhou and Y. Cui, Nat. Nanotechnol., 2018, 13, 294-299.

68 M. Wang, I. Al-Dhahir, J. Appiah and K. J. Koski, Chem. Mater., 2017, 29, 1650-1655.

69 J. P. Motter, K. J. Koski and Y. Cui, Chem. Mater., 2014, 26, 2313-2317.

70 M. Wang and K. J. Koski, ACS Nano, 2015, 9, 3226-3233.

71 N. C. Osti, M. Naguib, A. Ostadhossein, Y. Xie, P. R. Kent, B. Dyatkin, G. Rother, W. T. Heller, A. C. van Duin, Y. Gogotsi and E. Mamontov, ACS Appl. Mater. Interfaces, 2016, 8, 8859-8863.

72 O. Mashtalir, M. Naguib, V. N. Mochalin, Y. Dall'Agnese, M. Heon, M. W. Barsoum and Y. Gogotsi, Nat. Commun., 2013, 4, 1716.

73 O. Mashtalir, M. R. Lukatskaya, M.-Q. Zhao, M. W. Barsoum and Y. Gogotsi, Adv. Mater., 2015, 27, 3501-3506.

74 M. Naguib, M. Kurtoglu, V. Presser, J. Lu, J. Niu, M. Heon, L. Hultman, Y. Gogotsi and M. W. Barsoum, Adv. Mater., 2011, 23, 4248-4253.

75 J. Li, X. Yuan, C. Lin, Y. Yang, L. Xu, X. Du, J. Xie, J. Lin and J. Sun, Adv. Energy Mater., 2017, 7, 1602725.

76 J. L. Hart, K. Hantanasirisakul, A. C. Lang, B. Anasori, D. Pinto, Y. Pivak, J. T. van Omme, S. J. May, Y. Gogotsi and M. L. Taheri, Nat. Commun., 2019, 10, 522.

77 M. Boota, M. Pasini, F. Galeotti, W. Porzio, M.-Q. Zhao, J. Halim and Y. Gogotsi, Chem. Mater., 2017, 29, 2731-2738.

78 M. Okubo, A. Sugahara, S. Kajiyama and A. Yamada, Acc. Chem. Res., 2018, 51, 591-599.

79 L. Zhao, R. Zhang, C. Deng, Y. Peng and T. Jiang, Nanomaterials, 2019, 9, 1096.

80 N. I. Kovtyukhova, Y. Wang, R. Lv, M. Terrones, V. H. Crespi and T. E. Mallouk, J. Am. Chem. Soc., 2013, 135, 8372-8381.

81 S. Yang, P. Zhang, A. S. Nia and X. Feng, Adv. Mater., 2020, 32, 1907857.

82 J. Zhang, A. Yang, X. Wu, J. van de Groep, P. Tang, S. Li, B. Liu, F. Shi, J. Wan, Q. Li, Y. Sun, Z. Lu, X. Zheng, G. Zhou, C.-L. Wu, S.-C. Zhang, M. L. Brongersma, J. Li and Y. Cui, Nat. Commun., 2018, 9, 5289.

83 J. Cao, P. He, M. A. Mohammed, X. Zhao, R. J. Young, B. Derby, I. A. Kinloch and R. A. W. Dryfe, J. Am. Chem. Soc., 2017, 139, 17446-17456.

84 F. Xiong, H. Wang, X. Liu, J. Sun, M. Brongersma, E. Pop and Y. Cui, Nano Lett., 2015, 15, 6777-6784.

85 C. Wang, Q. He, U. Halim, Y. Liu, E. Zhu, Z. Lin, H. Xiao, X. Duan, Z. Feng, R. Cheng, N. O. Weiss, G. Ye, Y.-C. Huang, H. Wu, H.-C. Cheng, I. Shakir, L. Liao, X. Chen, W. A. Goddard III, Y. Huang and X. Duan, Nature, 2018, 555, 231-236.

86 L. Oakes, R. Carter, T. Hanken, A. P. Cohn, K. Share, B. Schmidt and C. L. Pint, Nat. Commun., 2016, 7, 11796.

87 D. K. Bediako, M. Rezaee, H. Yoo, D. T. Larson, S. Y. F. Zhao, T. Taniguchi, K. Watanabe, T. L. Brower-Thomas, E. Kaxiras and P. Kim, Nature, 2018, 558, 425-429.

88 Y. Li, H. Yan, B. Xu, L. Zhen and C.-Y. Xu, Adv. Mater., 2020, 2000581. 
89 E. R. White, J. J. Lodico and B. C. Regan, Nat. Commun., 2017, 8, 1969.

90 V. V. Avdeev, O. A. Tverezovskaya, N. E. Sorokina, I. V. Nikol'skaya and A. I. Finaenov, Inorg. Mater., 2000, 36, 214-218.

91 J. A. Seel and J. R. Dahn, J. Electrochem. Soc., 2000, 147, 892898.

92 K. Ji, J. Han, A. Hirata, T. Fujita, Y. Shen, S. Ning, P. Liu, H. Kashani, Y. Tian, Y. Ito, J.-I. Fujita and Y. Oyama, Nat. Commun., 2019, 10, 275.

93 M. Kühne, F. Paolucci, J. Popovic, P. M. Ostrovsky, J. Maier and J. H. Smet, Nat. Nanotechnol., 2017, 12, 895-900.

94 Q. He, Z. Lin, M. Ding, A. Yin, U. Halim, C. Wang, Y. Liu, H.-C. Cheng, Y. Huang and X. Duan, Nano Lett., 2019, 19, 6819-6826.

95 W. He, H. Zang, S. Cai, Z. Mu, C. Liu, M. Ding, P. Wang and X. Wang, Nano Res., 2020, 13, 2917-2924.

96 D. Wang, L.-M. Liu, S.-J. Zhao, Z.-Y. Hu and H. Liu, J. Phys. Chem. C, 2016, 120, 4779-4788.

97 Y. Cao, V. Fatemi, A. Demir, S. Fang, S. L. Tomarken, J. Y. Luo, J. D. Sanchez-Yamagishi, K. Watanabe, T. Taniguchi, E. Kaxiras, R. C. Ashoori and P. JarilloHerrero, Nature, 2018, 556, 80-84.

98 A. Kerelsky, L. J. McGilly, D. M. Kennes, L. Xian, M. Yankowitz, S. Chen, K. Watanabe, T. Taniguchi, J. Hone, C. Dean, A. Rubio and A. N. Pasupathy, Nature, 2019, 572, 95-100.

99 J. W. Jeon, H. Kim, H. Kim, S. Choi and B. H. Kim, AIP Adv., 2018, 8, 075228.

100 A. Black, J. Roberts, M. Acebrón, R. Bernardo-Gavito, G. Alsharif, F. J. Urbanos, B. H. Juárez, O. V. Kolosov, B. J. Robinson, R. Miranda, A. L. Vázquez de Parga, D. Granados and R. J. Young, ACS Appl. Mater. Interfaces, 2018, 10, 6805-6809.

101 R. Mirzayev, K. Mustonen, M. R. A. Monazam, A. Mittelberger, T. J. Pennycook, C. Mangler, T. Susi, J. Kotakoski and J. C. Meyer, Sci. Adv., 2017, 3, e1700176.

102 D. Deng, K. S. Novoselov, Q. Fu, N. Zheng, Z. Tian and X. Bao, Nat. Nanotechnol., 2016, 11, 218-230.

103 W. Zhang, Q. Wang, Y. Chen, Z. Wang and A. T. S. Wee, $2 D$ Mater., 2016, 3, 022001.

104 X. Zhou, X. Hu, J. Yu, S. Liu, Z. Shu, Q. Zhang, H. Li, Y. Ma, H. Xu and T. Zhai, Adv. Funct. Mater., 2018, 28, 1706587.

105 R. Frisenda, E. Navarro-Moratalla, P. Gant, D. Pérez de Lara, P. Jarillo-Herrero, R. V. Gorbachev and A. CastellanosGomez, Chem. Soc. Rev., 2018, 47, 53-68.

106 X. Yang and M. Yan, Nano Res., 2020, 13, 599-610.

107 J. W. Suk, A. Kitt, C. W. Magnuson, Y. Hao, S. Ahmed, J. An, A. K. Swan, B. B. Goldberg and R. S. Ruoff, ACS Nano, 2011, 5, 6916-6924.

108 Z. Hu, Z.-B. Liu and J.-G. Tian, Chin. J. Org. Chem., 2020, 38, 981-995.

109 X. Ma, Q. Liu, D. Xu, Y. Zhu, S. Kim, Y. Cui, L. Zhong and M. Liu, Nano Lett., 2017, 17, 6961-6967.

110 Y. Hou, X. Ren, J. Fan, G. Wang, Z. Dai, C. Jin, W. Wang, Y. Zhu, S. Zhang, L. Liu and Z. Zhang, ACS Appl. Mater. Interfaces, 2020, 12, 40958-40967.
111 Y. Huang, Y.-H. Pan, R. Yang, L.-H. Bao, L. Meng, H.-L. Luo, Y.-Q. Cai, G.-D. Liu, W.-J. Zhao, Z. Zhou, L.-M. Wu, Z.-L. Zhu, M. Huang, L.-W. Liu, L. Liu, P. Cheng, K.-H. Wu, S.-B. Tian, C.-Z. Gu, Y.-G. Shi, Y.-F. Guo, Z.-G. Cheng, J. P. Hu, L. Zhao, G.-H. Yang, E. Sutter, P. Sutter, Y.-L. Wang, W. Ji, X.-J. Zhou and H.-J. Gao, Nat. Commun., 2020, 11, 2453.

112 M. Hußmann, B. Weintrub, P. Feicht, G. Germer, J. N. Kirchhof, K. I. Bolotin and S. Eigler, Nanoscale Adv., 2020, 2, 176-181.

113 M. Velický, G. E. Donnelly, W. R. Hendren, S. McFarland, D. Scullion, W. J. I. DeBenedetti, G. C. Correa, Y. Han, A. J. Wain, M. A. Hines, D. A. Muller, K. S. Novoselov, H. D. Abruña, R. M. Bowman, E. J. G. Santos and F. Huang, ACS Nano, 2018, 12, 10463-10472.

114 G. F. Schneider, V. E. Calado, H. Zandbergen, L. M. K. Vandersypen and C. Dekker, Nano Lett., 2010, 10, 1912-1916.

115 J. G. Bednorz and K. A. Müller, Angew. Chem., Int. Ed., 1988, 27, 735-748.

116 G. F. Sun, K. W. Wong, B. R. Xu, Y. Xin and D. F. Lu, Phys. Lett. A, 1994, 192, 122-124.

117 Z. M. Geballe, H. Liu, A. K. Mishra, M. Ahart, M. Somayazulu, Y. Meng, M. Baldini and R. J. Hemley, Angew. Chem., Int. Ed., 2018, 130, 696-700.

118 I. A. Kruglov, D. V. Semenok, H. Song, R. Szczęśniak, I. A. Wrona, R. Akashi, E. M. M. Davari, D. Duan, T. Cui, A. G. Kvashnin and A. R. Oganov, Phys. Rev. B, 2020, 101, 024508.

119 A. F. Hebard, M. J. Rosseinsky, R. C. Haddon, D. W. Murphy, S. H. Glarum, T. T. M. Palstra, A. P. Ramirez and A. R. Kortan, Nature, 1991, 350, 600-601.

120 Z. K. Tang, L. Zhang, N. Wang, X. X. Zhang, G. H. Wen, G. D. Li, J. N. Wang, C. T. Chan and P. Sheng, Science, 2001, 292, 2462-2465.

121 E. A. Ekimov, V. A. Sidorov, E. D. Bauer, N. N. Mel'nik, N. J. Curro, J. D. Thompson and S. M. Stishov, Nature, 2004, 428, 542-545.

122 B. Uchoa and A. H. C. Neto, Phys. Rev. Lett., 2007, 98, 146801.

123 Y. Cao, V. Fatemi, S. Fang, K. Watanabe, T. Taniguchi, E. Kaxiras and P. Jarillo-Herrero, Nature, 2018, 556, 43-50.

124 S. Ichinokura, K. Sugawara, A. Takayama, T. Takahashi and S. Hasegawa, ACS Nano, 2016, 10, 2761-2765.

125 J.-H. Liao, Y.-C. Zhao, Y.-J. Zhao, H. Xu and X.-B. Yang, Phys. Chem. Chem. Phys., 2017, 19, 29237-29243.

126 K. S. Novoselov, A. K. Geim, S. V. Morozov, D. Jiang, Y. Zhang, S. V. Dubonos, I. V. Grigorieva and A. A. Firsov, Science, 2004, 306, 666-669.

127 W. Zhang, C.-T. Lin, K.-K. Liu, T. Tite, C.-Y. Su, C.-H. Chang, Y.-H. Lee, C.-W. Chu, K.-H. Wei, J.-L. Kuo and L.-J. Li, ACS Nano, 2011, 5, 7517-7524.

128 J. B. Oostinga, H. B. Heersche, X. Liu, A. F. Morpurgo and L. M. K. Vandersypen, Nat. Mater., 2008, 7, 151-157.

129 Y. Zhang, T.-T. Tang, C. Girit, Z. Hao, M. C. Martin, A. Zettl, M. F. Crommie, Y. R. Shen and F. Wang, Nature, 2009, 459, 820-823. 
130 H. Guo, R. Zhang, H. Li, X. Wang, H. Lu, K. Qian, G. Li, L. Huang, X. Lin, Y.-Y. Zhang, H. Ding, S. Du, S. T. Pantelides and H.-J. Gao, Nano Lett., 2020, 20, 26742680.

131 D. Zhan, J. X. Yan, Z. H. Ni, L. Sun, L. F. Lai, L. Liu, X. Y. Liu and Z. X. Shen, Small, 2015, 11, 1177-1182.

132 J. W. Yang, G. Lee, J. S. Kim and K. S. Kim, J. Phys. Chem. Lett., 2011, 2, 2577-2581.

133 R. Schlögl, W. Jones and H.-P. Boehm, Synth. Met., 1983, 7, 133-140.

134 K. Li, T. H. Chang, Q. Xie, Y. Cheng, H. Yang, J. Chen and P. Y. Chen, Adv. Electron. Mater., 2019, 5, 1900040.

135 S. Yang, L. Chen, L. Mu and P.-C. Ma, J. Colloid Interface Sci., 2014, 430, 337-344.

136 K. Yang, L. Feng and Z. Liu, Adv. Drug Delivery Rev., 2016, 105, 228-241.

137 X. Lu, X. Feng, J. R. Werber, C. Chu, I. Zucker, J.-H. Kim, C. O. Osuji and M. Elimelech, Proc. Natl. Acad. Sci. U. S. A., 2017, 114, E9793-E9801.

138 X. Zhang, X. Zhao and Y. Liu, J. Phys. Chem. C, 2016, 120, 22710-22717.

139 M. Kinany-Alaoui, L. Piraux, V. Bayot, J.-P. Issi, P. Pernot and R. Vangélisti, Synth. Met., 1989, 34, 537-542.

140 K. Sugihara, N.-C. Yeh, M. S. Dresselhaus and G. Dresselhaus, Phys. Rev. B: Condens. Matter Mater. Phys., 1989, 39, 4577-4587.

141 Z. Li, X. Zhang, X. Zhao, J. Li, T. S. Herng, H. Xu, F. Lin, P. Lyu, X. Peng, W. Yu, X. Hai, C. Chen, H. Yang, J. Martin, J. Lu, X. Luo, A. H. Castro Neto, S. J. Pennycook, J. Ding, Y. Feng and J. Lu, Adv. Mater., 2020, 32, 1907645.

142 E. Coronado, C. Martí-Gastaldo, E. Navarro-Moratalla, A. Ribera, S. J. Blundell and P. J. Baker, Nat. Chem., 2010, 2, 1031-1036.

143 B. Baek, W. H. Rippard, S. P. Benz, S. E. Russek and P. D. Dresselhaus, Nat. Commun., 2014, 5, 3888.

144 S. Tongay, K. Berke, M. Lemaitre, Z. Nasrollahi, D. B. Tanner, A. F. Hebard and B. R. Appleton, Nanotechnology, 2011, 22, 425701.

145 T.-H. Han, Y. Lee, M.-R. Choi, S.-H. Woo, S.-H. Bae, B. H. Hong, J.-H. Ahn and T.-W. Lee, Nat. Photonics, 2012, 6, 105-110.

146 F. Güneș, H.-J. Shin, C. Biswas, G. H. Han, E. S. Kim, S. J. Chae, J.-Y. Choi and Y. H. Lee, ACS Nano, 2010, 4, 4595-4600.

147 G. R. Hennig, J. Chem. Phys., 1965, 43, 1201-1206.
148 W. Bao, J. Wan, X. Han, X. Cai, H. Zhu, D. Kim, D. Ma, Y. Xu, J. N. Munday, H. D. Drew, M. S. Fuhrer and L. Hu, Nat. Commun., 2014, 5, 4224.

149 N. A. W. Holzwarth, S. G. Louie and S. Rabii, Phys. Rev. B: Condens. Matter Mater. Phys., 1983, 28, 1013-1025.

150 T. Mueller, F. Xia and P. Avouris, Nat. Photonics, 2010, 4, 297-301.

151 H. Zhang, ACS Nano, 2015, 9, 9451-9469.

152 F. Bonaccorso, Z. Sun, T. Hasan and A. C. Ferrari, Nat. Photonics, 2010, 4, 611-622.

153 M. Freitag, T. Low, F. Xia and P. Avouris, Nat. Photonics, 2012, 7, 53-59.

154 R. R. Nair, P. Blake, A. N. Grigorenko, K. S. Novoselov, T. J. Booth, T. Stauber, N. M. Peres and A. K. Geim, Science, 2008, 320, 1308.

155 M. Liu, X. Yin, E. Ulin-Avila, B. Geng, T. Zentgraf, L. Ju, F. Wang and X. Zhang, Nature, 2011, 474, 64-67.

156 Z. Fang, Z. Liu, Y. Wang, P. M. Ajayan, P. Nordlander and N. J. Halas, Nano Lett., 2012, 12, 3808-3813.

157 Y. Lee, H. Kim, S. Kim, D. Whang and J. H. Cho, ACS Appl. Mater. Interfaces, 2019, 11, 23474-23481.

158 Q. Nian, L. Gao, Y. Hu, B. Deng, J. Tang and G. J. Cheng, ACS Appl. Mater. Interfaces, 2017, 9, 44715-44723.

159 J. Kim, C. Jin, B. Chen, H. Cai, T. Zhao, P. Lee, S. Kahn, K. Watanabe, T. Taniguchi, S. Tongay, M. F. Crommie and F. Wang, Sci. Adv., 2017, 3, e1700518.

160 H. Zhou, Y. Zhao, W. Tao, Y. Li, Q. Zhou and H. Zhu, ACS Nano, 2020, 14, 4618-4625.

161 P. Zielinski, M. Kühne, D. Kärcher, F. Paolucci, P. Wochner, S. Fecher, J. Drnec, R. Felici and J. H. Smet, Nano Lett., 2019, 19, 3634-3640.

162 F. Li, J. Zou, L. Cao, Z. Li, S. Gu, Y. Liu, J. Zhang, H. Liu and Z. Lu, J. Phys. Chem. C, 2019, 123, 5067-5072.

163 J. P. Bonacum, A. O'Hara, D.-L. Bao, O. S. Ovchinnikov, Y.-F. Zhang, G. Gordeev, S. Arora, S. Reich, J.-C. Idrobo, R. F. Haglund, S. T. Pantelides and K. I. Bolotin, Phys. Rev. Mater., 2019, 3, 064004.

164 P. Xiong, F. Zhang, X. Zhang, S. Wang, H. Liu, B. Sun, J. Zhang, Y. Sun, R. Ma, Y. Bando, C. Zhou, Z. Liu, T. Sasaki and G. Wang, Nat. Commun., 2020, 11, 3297.

165 E. Pomerantseva and Y. Gogotsi, Nat. Energy, 2017, 2, 17089.

166 P. A. Vermeulen, J. Mulder, J. Momand and B. J. Kooi, Nanoscale, 2018, 10, 1474-1480.

167 G. Algara-Siller, S. Kurasch, M. Sedighi, O. Lehtinen and U. Kaiser, Appl. Phys. Lett., 2013, 103, 203107.

168 M.-H. Huang, X.-C. Dai, T. Li, Y.-B. Li, Y. He, G. Xiao and F.-X. Xiao, J. Phys. Chem. C, 2019, 123, 9721-9734. 